\title{
Recursive formulation of the multiconfigurational time-dependent Hartree method for fermions, bosons and mixtures thereof in terms of one-body density operators
}

\author{
Ofir E. Alon ${ }^{1 *}$, Alexej I. Streltsov ${ }^{2 \dagger}$, Kaspar Sakmann ${ }^{2 \ddagger}$, \\ Axel U. J. Lode ${ }^{2 \S}$, Julian Grond ${ }^{2 \uparrow}$, and Lorenz S. Cederbaum² ${ }^{2 \|}$ \\ ${ }^{1}$ Department of Physics, University of Haifa at Oranim, Tivon 36006, Israel. and \\ 2 Theoretische Chemie, Physikalisch-Chemisches Institut, Universität Heidelberg, \\ Im Neuenheimer Feld 229, D-69120 Heidelberg, Germany.
}

\footnotetext{
* ofir@research.haifa.ac.il

$\dagger$ alexej.streltsov@pci.uni-heidelberg.de

‡ kaspar.sakmann@pci.uni-heidelberg.de

$\S$ axel.lode@pci.uni-heidelberg.de

ฯ julian.grond@pci.uni-heidelberg.de

I| lorenz.cederbaum@pci.uni-heidelberg.de
} 


\begin{abstract}
The multiconfigurational time-dependent Hartree method (MCTDH) [H.-D. Meyer, U. Manthe, and L. S. Cederbaum, Chem. Phys. Lett. 165, 73 (1990); U. Manthe, H.-D. Meyer, and L. S. Cederbaum, J. Chem. Phys. 97, 3199 (1992)] is celebrating nowadays entering its third decade of tackling numerically-exactly a broad range of correlated multi-dimensional non-equilibrium quantum dynamical systems. Taking in recent years particles' statistics explicitly into account, within the MCTDH for fermions (MCTDHF) and for bosons (MCTDHB), has opened up further opportunities to treat larger systems of interacting identical particles, primarily in laser-atom and cold-atom physics. With the increase of experimental capabilities to simultaneously trap mixtures of two, three, and possibly even multiple kinds of interacting composite identical particles together, we set up the stage in the present work and specify the MCTDH method for such cases. Explicitly, the MCTDH method for systems with three kinds of identical particles interacting via all combinations of two- and three-body forces is presented, and the resulting equations-of-motion are briefly discussed. All four possible mixtures (Fermi-Fermi-Fermi, Bose-Fermi-Fermi, Bose-Bose-Fermi and Bose-Bose-Bose) are presented in a unified manner. Particular attention is paid to represent the coefficients' part of the equations-of-motion in a compact recursive form in terms of one-body density operators only. The recursion utilizes the recently proposed Combinadic-based mapping for fermionic and bosonic operators in Fock space [A. I. Streltsov, O. E. Alon, and L. S. Cederbaum, Phys. Rev. A 81, 022124 (2010)] and successfully applied and implemented within MCTDHB. Our work sheds new light on the representation of the coefficients' part in MCTDHF and MCTDHB without resorting to the matrix elements of the many-body Hamiltonian with respect to the timedependent configurations. It suggests a recipe for efficient implementation of the schemes derived here for mixtures which is suitable for parallelization.
\end{abstract}

PACS numbers: 31.15.xv, 67.60.-g, 05.30.Fk, 05.30.Jp, 03.65.-w 


\section{INTRODUCTION}

Quantum non-equilibrium dynamics is important to many branches of physics and chemistry $[1-6]$ and often requires the solution of the time-dependent many-particle Schrödinger equation. A particular efficient method to solve the time-dependent manyparticle Schrödinger equation is the multiconfigurational time-dependent Hartree (MCTDH) algorithm and approach [7-10]. MCTDH, which is considered at present the most efficient wave-packet propagation tool, has amply been employed for multi-dimensional dynamical systems of distinguishable degrees-of-freedom, typically molecular vibrations, see, e.g., Refs. [11-18]. We mention that recent developments on multi-layer formulation of MCTDH have opened up further possibilities to treat larger systems of distinguishable degrees-offreedom [19-21]. MCTDH has recently been applied with much success to various systems with a few identical particles in the field of cold-atom physics, see, e.g., Refs. [22-27].

In recent years, taking the quantum statistics between identical particles a priori into account, the MCTDH method has been specified for systems of identical particles, which opened up interesting possibilities to treat larger systems. First MCTDHF - the fermionic version of MCTDH - was developed by three independent groups [28-30]. Shortly after, MCTDHB - the bosonic version of MCTDH - was developed in [31, 32]. For applications of MCTDHF to laser-matter interaction and other few-fermion problems see, e.g., Refs. [3342], where the last work combines optimal control theory with MCTDHF. For applications of MCTDHB to Bose-Einstein condensates see, e.g., Refs. [43-47], where the last two works combine optimal control theory with MCTDHB.

Since the seminal paper of Löwdin [48], reduced density matrices and particularly reduced two-body density matrices have been a lively field of research, see, e.g., Refs. [49-55]. Reduced one-body density matrices are an inherent part of the $\mathrm{MCTDH}$ [7-10]. In the present context, reduced one- and two-body density matrices were first used to derive the static self-consistent theory for bosons, the multiconfigurational Hartree for bosons (MCHB) in [56]. Thereafter, MCTDHB and MCTDHF were formulated in a unified manner by employing reduced one-, two- [57] and three-body [10] density matrices. Further specification

of MCTDH to mixtures of two kinds of identical particles (MCTDH-FF for Fermi-Fermi mixtures; MCTDH-BF for Bose-Fermi mixtures; and MCTDH-BB for Bose-Bose mixtures) was put forward in [58]. All the above developments made use of the fact that the mean- 
field operators in the traditional MCTDH can be factorized to products of reduced density matrices times one-body operators. Finally, we mention that MCTDH has been extended to systems with particle conversion (termed MCTDH-conversion), where particles of one kind can convert to another kind [59].

A breakthrough in the formulation [60,61] and implementation [62] of MCTDHB has stemmed from a general Combinadic-based mapping of bosonic (and fermionic) operators in Fock space. In this formulation, the direct calculation of the matrix representation of the Hamiltonian in the (huge) multiconfigurational space is abandoned, and is replaced by the action of one-body and two-body density operators on the multiconfigurational wavefunction. The operation of the various density operators can be performed in parallel [62], which further accelerates the performance of the algorithm. This brings us closer to the topic and contents of the present work.

Two-body interaction is the most basic interaction in an interacting (quantum) system. When the particles comprising the quantum system have internal structure, higher-order interactions (forces) may come into play. For instance, in nuclear physics it has long been accepted that three-body interactions are necessary to fully understand the structure of nuclei, see, e.g. [63, 64]. Much more recently, and in the context of another field, the proposition to utilize cold polar molecules to engineer (condensed-matter) systems with three-body interactions has been made [65]. So, the motivation to study the non-equilibrium dynamics of systems with up to three-body forces is clear.

But why study the quantum dynamics of a mixture of three kinds of identical particles? Are such systems present in nature? In the cold-atom world, the plurality of atoms is one of the most important ingredients experimentalists (and theorists) have at their disposal. For instance, the element $\mathrm{Yb}$ has seven stable isotopes (5 bosonic and 2 fermionic isotopes). $\mathrm{Yb}$ has been envisaged to play an instrumental role in realizing various interesting ultra-cold mixtures (see Ref. [66] for a realization of a Bose-Einstein condensate with ${ }^{170} \mathrm{Yb}$ atoms and the discussion therein). More recently, a quantum degenerate Fermi-Fermi mixture of ${ }^{6} \mathrm{Li}^{4}{ }^{40} \mathrm{~K}$ atoms coexisting with a Bose-Einstein Condensate of ${ }^{87} \mathrm{Rb}$ atoms were realized [67], as well as a triply quantum-degenerate mixture of bosonic ${ }^{41} \mathrm{~K}$ atoms and two fermionic ${ }^{40} \mathrm{~K}$ and ${ }^{6} \mathrm{Li}$ atoms [68]. Hence, mixtures of three kinds of identical particles have been created in the lab.

All the above dictate the purposes and contents of the present work. The MCTDH 
method for systems with three kinds of identical particles interacting via all combinations of two- and three-body forces is derived, and the resulting equations-of-motion are briefly discussed. All four possible mixtures (Fermi-Fermi-Fermi, Bose-Fermi-Fermi, Bose-BoseFermi and Bose-Bose-Bose) are presented in a unified manner. Particular attention is paid to representing the coefficients' part of the equations-of-motion in a compact recursive form in terms of one-body density operators only. The recursion utilizes the recently proposed Combinadic-based mapping [60] which has already been successfully applied and implemented within MCTDHB [62]. Our work sheds new light on the representation of the coefficients' part in MCTDHF and MCTDHB without resorting to the matrix elements of the many-body Hamiltonian with respect to the time-dependent configurations, and suggests a recipe for efficient implementation of the theory derived here for mixtures which is suitable for parallelization.

The structure of the paper is as follows. In Sec. II we present the building bricks of the theory by reconstructing MCTDHF and MCTDHB. In Sec. III we assemble from these ingredients the multiconfigurational time-dependent Hartree method for mixtures of three kinds of identical particles interacting via up to three-body forces. A brief summary and outlook are given in Sec. IV. Finally, we collect in Appendixes A-C for completeness and ease of presentation of the main text various quantities appearing and needed in the derivation. The paper and the Appendixes are detailed and intended also to serve as a guide for the implementation of the equations-of-motion. The reconstruction of MCTDHF and MCTDHB is given in sufficient detail. This allows us to defer to the Appendixes much of the lengthly formulas used later on for the mixtures. 


\section{BUILDING BRICKS: RECONSTRUCTING MCTDHF AND MCTDHB}

\section{A. From basic ingredients to mapping}

Our starting point is the many-body Hamiltonian of $N_{A}$ interacting identical particles of type $A$ :

$$
\begin{aligned}
& \hat{H}^{(A)}=\hat{h}^{(A)}+\hat{W}^{(A)}+\hat{U}^{(A)}=\int d \mathbf{x}\left\{\hat{\mathbf{\Psi}}_{A}^{\dagger}(\mathbf{x}) \hat{h}^{(A)}(\mathbf{x}) \hat{\mathbf{\Psi}}_{A}(\mathbf{x})+\right. \\
+ & \frac{1}{2} \int d \mathbf{x}^{\prime}\left[\hat{\mathbf{\Psi}}_{A}^{\dagger}(\mathbf{x}) \hat{\mathbf{\Psi}}_{A}^{\dagger}\left(\mathbf{x}^{\prime}\right) \hat{W}^{(A)}\left(\mathbf{x}, \mathbf{x}^{\prime}\right) \hat{\mathbf{\Psi}}_{A}\left(\mathbf{x}^{\prime}\right) \hat{\mathbf{\Psi}}_{A}(\mathbf{x})+\right. \\
+ & \left.\left.\frac{1}{3} \int d \mathbf{x}^{\prime \prime} \hat{\mathbf{\Psi}}_{A}^{\dagger}(\mathbf{x}) \hat{\mathbf{\Psi}}_{A}^{\dagger}\left(\mathbf{x}^{\prime}\right) \hat{\mathbf{\Psi}}_{A}^{\dagger}\left(\mathbf{x}^{\prime \prime}\right) \hat{W}^{(A)}\left(\mathbf{x}, \mathbf{x}^{\prime}, \mathbf{x}^{\prime \prime}\right) \hat{\mathbf{\Psi}}_{A}\left(\mathbf{x}^{\prime \prime}\right) \hat{\mathbf{\Psi}}_{A}\left(\mathbf{x}^{\prime}\right) \hat{\mathbf{\Psi}}_{A}(\mathbf{x})\right]\right\}
\end{aligned}
$$

where $\hat{h}^{(A)}$ is the one-body part, $\hat{W}^{(A)}$ the two-body part and $\hat{U}^{(A)}$ the three-body part. The operators $\hat{h}^{(A)}, \hat{W}^{(A)}$ and $\hat{U}^{(A)}$ can generally be time-dependent.

We use the time-independent field operator expanded by time-dependent orbitals:

$$
\hat{\mathbf{\Psi}}_{A}(\mathbf{x})=\sum_{k} \hat{a}_{k}(t) \phi_{k}(\mathbf{x}, t)
$$

where the annihilation and creation operators obey the usual fermionic/bosonic anti/commutation relations, $\hat{a}_{q}(t) \hat{a}_{k}^{\dagger}(t) \pm \hat{a}_{k}^{\dagger}(t) \hat{a}_{q}(t)=\delta_{k q}$. Correspondingly, the field operator

obeys the anti/commutation relations, $\hat{\mathbf{\Psi}}_{A}(\mathbf{x})\left\{\hat{\mathbf{\Psi}}_{A}\left(\mathbf{x}^{\prime}\right)\right\}^{\dagger} \pm\left\{\hat{\mathbf{\Psi}}_{A}\left(\mathbf{x}^{\prime}\right)\right\}^{\dagger} \hat{\mathbf{\Psi}}_{A}(\mathbf{x})=\delta\left(\mathbf{x}-\mathbf{x}^{\prime}\right)$. Here and hereafter the upper sign refers to fermions and the lower to bosons. The coordinate $\mathbf{x} \equiv\{\mathbf{r}, \sigma\}$ stands for spatial degrees of freedom and spin, if present. Thus, the shorthand notations $\delta\left(\mathbf{x}-\mathbf{x}^{\prime}\right)=\delta\left(\mathbf{r}-\mathbf{r}^{\prime}\right) \delta_{\sigma, \sigma^{\prime}}$ and $\int d \mathbf{x} \equiv \int d \mathbf{r} \sum_{\sigma}$ are implied throughout this work. Furthermore, we do not denote explicitly the dependence of quantities on time when unambiguous.

Plugging the expansion (2) into the many-body Hamiltonian (1) one gets:

$$
\hat{H}^{(A)}=\sum_{k, q} h_{k q}^{(A)} \hat{\rho}_{k q}^{(A)}+\frac{1}{2} \sum_{k, s, q, l} W_{k s q l}^{(A)} \hat{\rho}_{k s l q}^{(A)}+\frac{1}{6} \sum_{k, s, p, r, l, q} U_{k s p q l}^{(A)} \hat{\rho}_{k s p r l q}^{(A)},
$$

where the matrix elements with respect to the orbitals $\left\{\phi_{k}(\mathbf{x}, t)\right\}$ are given by:

$$
\begin{aligned}
h_{k q}^{(A)} & =\int \phi_{k}^{*}(\mathbf{x}, t) \hat{h}^{(A)}(\mathbf{x}) \phi_{q}(\mathbf{x}, t) d \mathbf{x} \\
W_{k s q l}^{(A)} & =\iint \phi_{k}^{*}(\mathbf{x}, t) \phi_{s}^{*}\left(\mathbf{x}^{\prime}, t\right) \hat{W}^{(A)}\left(\mathbf{x}, \mathbf{x}^{\prime}\right) \phi_{q}(\mathbf{x}, t) \phi_{l}\left(\mathbf{x}^{\prime}, t\right) d \mathbf{x} d \mathbf{x}^{\prime} \\
U_{k s p q l r}^{(A)} & =\iiint \phi_{k}^{*}(\mathbf{x}, t) \phi_{s}^{*}\left(\mathbf{x}^{\prime}, t\right) \phi_{p}^{*}\left(\mathbf{x}^{\prime \prime}, t\right) \hat{U}^{(A)}\left(\mathbf{x}, \mathbf{x}^{\prime}, \mathbf{x}^{\prime \prime}\right) \times \\
& \times \phi_{q}(\mathbf{x}, t) \phi_{l}\left(\mathbf{x}^{\prime}, t\right) \phi_{r}\left(\mathbf{x}^{\prime \prime}, t\right) d \mathbf{x} d \mathbf{x}^{\prime} d \mathbf{x}^{\prime \prime}
\end{aligned}
$$


In (3), we introduce the one-body density operators

$$
\hat{\rho}_{k q}^{(A)}=\hat{a}_{k}^{\dagger} \hat{a}_{q}
$$

as well as the two- and three-body density operators

$$
\begin{aligned}
& \hat{\rho}_{k s l q}^{(A)}=\hat{a}_{k}^{\dagger} \hat{a}_{s}^{\dagger} \hat{a}_{l} \hat{a}_{q}= \pm \hat{\rho}_{k q}^{(A)} \delta_{s l} \mp \hat{\rho}_{k l}^{(A)} \hat{\rho}_{s q}^{(A)}, \\
& \hat{\rho}_{k s p r l q}^{(A)}=\hat{a}_{k}^{\dagger} \hat{a}_{s}^{\dagger} \hat{a}_{p}^{\dagger} \hat{a}_{r} \hat{a}_{l} \hat{a}_{q}= \pm \hat{\rho}_{k s l q}^{(A)} \delta_{p r}-\hat{\rho}_{k s r q}^{(A)} \delta_{p l}+\hat{\rho}_{k s r l}^{(A)} \hat{\rho}_{p q}^{(A)} .
\end{aligned}
$$

The reason for this choice of notation with density operators in (3) will become clear below. We see that the two-body density operators $\left\{\hat{\rho}_{k s l q}^{(A)}\right\}$ can be written as products of the onebody density operators, and that the three-body density operators $\left\{\hat{\rho}_{k s p r l q}^{(A)}\right\}$ can be written as products of the two- and one-body density operators, and so on, recursively. Hence, the one-body density operators $\left\{\hat{\rho}_{k q}^{(A)}\right\}$ in (5) are our basic building bricks.

The many-body wave-function is expanded by time-dependent configurations (determinants $|\mathbf{i} ; t\rangle$ for fermions, permanents $|\mathbf{n} ; t\rangle$ for bosons) assembled by distributing the $N_{A}$ particles over the $M_{A}$ time-dependent orbitals introduced in the expansion (2). For fermions we write [60]:

$$
\left|\Psi^{(A)}(t)\right\rangle=\sum_{\{\mathbf{i}\}} C_{\mathbf{i}}(t)|\mathbf{i} ; t\rangle \equiv \sum_{J_{A}=1}^{N_{\text {conf }}^{(A)}} C_{J_{A}}(t)\left|J_{A} ; t\right\rangle,
$$

where the address $J_{A}$ is defined as follows:

$$
J_{A} \equiv J_{A}(\mathbf{i})=1+\sum_{j=1}^{M_{A}-N_{A}}\left(\begin{array}{c}
M_{A}-i_{j} \\
M_{A}-N_{A}+1-j
\end{array}\right)
$$

whereas for bosons we write [60]:

$$
\left|\Psi^{(A)}(t)\right\rangle=\sum_{\{\mathbf{n}\}} C_{\mathbf{n}}(t)|\mathbf{n} ; t\rangle \equiv \sum_{J_{A}=1}^{N_{c o n f}^{(A)}} C_{J_{A}}(t)\left|J_{A} ; t\right\rangle
$$

where the address $J_{A}$ is defined as follows:

$$
J_{A} \equiv J_{A}(\mathbf{n})=1+\sum_{k=1}^{M_{A}-1}\left(\begin{array}{c}
N_{A}+M_{A}-1-k-\sum_{l=1}^{k} n_{l} \\
M_{A}-k
\end{array}\right) .
$$

The notation used in (7-10) follows the Combinadic-based addressing scheme of configurations introduced in [60]. For fermions we enumerate configurations by holes, $\mathbf{i}=$ $\left(i_{1}, i_{2}, \ldots, i_{j}=q, \ldots, i_{M_{A}-N_{A}}\right)$ and $\mathbf{i}^{k q}=\left(i_{1}, i_{2}, \ldots, i_{l}=k, \ldots, i_{M_{A}-N_{A}}\right)$, whereas for 
bosons we enumerate configurations by particles, $\mathbf{n}=\left(n_{1}, \ldots, n_{k}, \ldots, n_{q}, \ldots, n_{M_{A}}\right)$ and $\mathbf{n}^{k q}=\left(n_{1}, \ldots, n_{k}-1, \ldots, n_{q}+1, \ldots, n_{M_{A}}\right)$. The index $J_{A}$ is termed "address" because it is an integer uniquely identifying a configuration which is described by the positions of the holes $\mathbf{i}$ (for fermions) or the occupation numbers $\mathbf{n}$ (for bosons). For more details of the Combinadic-based mapping and particularly the connection between the bosonic occupation numbers and the positions of the fermionic holes see [60].

For our requirements, we will need the result of the operation of the basic building bricks onto the state vector, namely, the operation of the one-body density operators $\left\{\hat{\rho}_{k q}^{(A)}\right\}$ onto $\left|\Psi^{(A)}(t)\right\rangle$. Thus we have:

$$
\hat{\rho}_{k q}^{(A)}\left|\Psi^{(A)}(t)\right\rangle=\hat{\rho}_{k q}^{(A)} \sum_{J_{A}=1}^{N_{c o n f}^{(A)}} C_{J_{A}}(t)\left|J_{A} ; t\right\rangle \equiv \sum_{J_{A}=1}^{N_{c o n f}^{(A)}} C_{J_{A}}^{\hat{\rho}_{k q}^{(A)}}(t)\left|J_{A} ; t\right\rangle .
$$

For fermions we have the following relations [60]:

$$
C_{J_{A}}^{\hat{\rho}_{k q}^{(A)}}(t) \equiv C_{J_{A}(\mathbf{i})}^{\hat{\rho}_{k q}^{(A)}}(t)=\left\{\begin{array}{cc}
C_{J_{A}\left(\mathbf{i} \mathbf{i}^{k q}\right)}(t) \times(-1)^{d\left(\mathbf{i}^{k q}\right)} ; & k \neq q, k \in \mathbf{i}^{k q}, q \notin \mathbf{i}^{k q} \\
C_{J_{A}(\mathbf{i})}(t) ; & k=q, k \notin \mathbf{i} \\
0 ; & \text { otherwise }
\end{array}\right.
$$

where the distance between the $i_{j}$-th hole of $\mathbf{i}$ at orbital $q$ and the $i_{l}$-th hole of $\mathbf{i}^{k q}$ at orbital $k$ is given by $d\left(\mathbf{i}^{k q}\right)=|k-q|-|j-l|-1$ [equivalently, $d\left(\mathbf{i}^{k q}\right)=\sum_{p \in(k, q)} n_{p}$ simply enumerates how many fermions are there between the $k$-th and $q$-th orbitals]. For bosons we have the following relations [60]:

$$
C_{J_{A}}^{\hat{\rho}_{k q}^{(A)}}(t) \equiv C_{J_{A}(\mathbf{n})}^{\hat{\rho}_{k q}^{(A)}}(t)=\left\{\begin{array}{cl}
C_{J_{A}\left(\mathbf{n}^{k q}\right)}(t) \times \sqrt{n_{k}} \sqrt{n_{q}+1} ; & k \neq q \\
C_{J_{A}(\mathbf{n})}(t) \times n_{k} ; & k=q
\end{array}\right.
$$

which concludes our exposition of the Combinadic-based mapping and assembly of the operations of the basic building bricks $\left\{\hat{\rho}_{k q}^{(A)}\right\}$ on the many-body wave-function $\left|\Psi^{(A)}(t)\right\rangle$. From Eqs. $(5,6)$ we see how to use the one-body (basic) building bricks $\left\{\hat{\rho}_{k q}^{(A)}\right\}$ to assemble higher-body operators. In particular we find:

$$
\begin{aligned}
& C_{J_{A}}^{\hat{\rho}_{k s l q}^{(A)}}(t)= \pm \delta_{s l} C_{J_{A}}^{\hat{\rho}_{k q}^{(A)}}(t) \mp C_{J_{A}}^{\hat{\rho}_{s q}^{(A)} \hat{\rho}_{k l}^{(A)}}(t) \\
& C_{J_{A}}^{\hat{\rho}_{k s p r l q}^{(A)}}(t)= \pm \delta_{p r} C_{J_{A}}^{\hat{\rho}_{k s l q}^{(A)}}(t)-\delta_{p l} C_{J_{A}}^{\hat{\rho}_{k s r q}^{(A)}}(t)+C_{J_{A}}^{\hat{\rho}_{p q}^{(A)} \hat{\rho}_{k s r l}^{(A)}}(t)
\end{aligned}
$$

The meaning of the two levels of density operators in the superscripts of the coefficients $C_{J_{A}}^{\hat{\rho}_{k s l q}^{(A)}}(t)$ and $C_{J_{A}}^{\hat{\rho}_{k s p r l q}^{(A)}}(t)$, resulting from higher-body operators in (14), is that the lower-level 
density operator is multiplied on the many-body wave-function first, and the upper-level density operator is multiplied thereafter on the result.

The key ingredient in the utilization of the Lagrangian formulation [32, 69, 70] of the (Dirac-Frenkel [71, 72]) time-dependent variational principle to derive the equations-ofmotion is the evaluation of matrix elements with respect to the multiconfigurational wavefunction $\left|\Psi^{(A)}(t)\right\rangle$. This will be utilized in the next subsection IIB. For the moment, we would like to prescribe how such matrix elements with respect to $\left|\Psi^{(A)}(t)\right\rangle$ are to be evaluated.

Consider the operator $\hat{O}^{(A)}$, which can be a one-body operator, two-body operator, threebody operator, etc. Then, we express and compute the expectation value of $\hat{O}^{(A)}$ with respect to $\left|\Psi^{(A)}(t)\right\rangle$ as follows $[60]$ :

$$
\left\langle\Psi^{(A)}(t)\left|\hat{O}^{(A)}\right| \Psi^{(A)}(t)\right\rangle=\left\langle\Psi^{(A)}(t)\right|\left\{\hat{O}^{(A)}\left|\Psi^{(A)}(t)\right\rangle\right\}=\sum_{J_{A}=1}^{N_{c o n f}^{(A)}} C_{J_{A}}^{*}(t) C_{J_{A}}^{\hat{O}^{(A)}}(t),
$$

where

$$
\hat{O}^{(A)}\left|\Psi^{(A)}(t)\right\rangle=\hat{O}^{(A)} \sum_{J_{A}=1}^{N_{\text {conf }}^{(A)}} C_{J_{A}}(t)\left|J_{A} ; t\right\rangle \equiv \sum_{J_{A}=1}^{N_{\text {conf }}^{(A)}} C_{J_{A}}^{\hat{O}^{(A)}}(t)\left|J_{A} ; t\right\rangle .
$$

In particular, for a one-body operator, $\hat{O}^{(A)}=\sum_{k, q} O_{k q}^{(A)} \hat{\rho}_{k q}^{(A)}$, we get:

$$
C_{J_{A}}^{\hat{O}^{(A)}}(t)=\sum_{k, q}^{M_{A}} O_{k q}^{(A)} C_{J_{A}}^{\hat{\rho}_{k q}^{(A)}}(t)
$$

for a two-body operator, $\hat{O}^{(A)}=\frac{1}{2} \sum_{k, s, q, l} O_{k s q l}^{(A)} \hat{\rho}_{k s l q}^{(A)}$, we get from (14):

$$
\begin{aligned}
C_{J_{A}}^{\hat{O}^{(A)}}(t) & =\frac{1}{2} \sum_{k, s, q, l}^{M_{A}} O_{k s q l}^{(A)} C_{J_{A}}^{\hat{\rho}_{k s l q}^{(A)}}(t)= \\
& =\frac{1}{2} \sum_{k, s, q, l}^{M_{A}} O_{k s q l}^{(A)}\left[ \pm \delta_{s l} C_{J_{A}}^{\hat{\rho}_{k q}^{(A)}}(t) \mp C_{J_{A}}^{\hat{\rho}_{s q}^{(A)} \hat{\rho}_{k l}^{(A)}}(t)\right]
\end{aligned}
$$

and for a three-body operator, $\hat{O}^{(A)}=\frac{1}{6} \sum_{k, s, p, r, l, q} O_{k s p q l r}^{(A)} \hat{\rho}_{k s p r l q}^{(A)}$, we get from (14):

$$
\begin{gathered}
C_{J_{A}}^{\hat{O}^{(A)}}(t)=\frac{1}{6} \sum_{k, s, p, r, l, q}^{M_{A}} O_{k s p q l r}^{(A)} C_{J_{A}}^{\hat{\rho}_{k s p r l q}^{(A)}}(t)= \\
=\frac{1}{6} \sum_{k, s, p, r, l, q}^{M_{A}} O_{k s p q l r}^{(A)}\left[ \pm \delta_{p r} C_{J_{A}}^{\hat{\rho}_{k s l q}^{(A)}}(t)-\delta_{p l} C_{J_{A}}^{\hat{\rho}_{k s q}^{(A)}}(t)+C_{J_{A}}^{\hat{\rho}_{\rho q}^{(A)} \hat{\rho}_{k s r l}^{(A)}}(t)\right] .
\end{gathered}
$$


Finally and generally, the result of a sum of (operations of) operators, e.g., $\hat{O}_{1}^{(A)}+\hat{O}_{2}^{(A)}$, on $\left|\Psi^{(A)}(t)\right\rangle$ translates to the sum of the respective coefficients [60]:

$$
C_{J_{A}}^{\hat{O}_{1}^{(A)}+\hat{O}_{2}^{(A)}}(t)=C_{J_{A}}^{\hat{O}_{1}^{(A)}}(t)+C_{J_{A}}^{\hat{O}_{2}^{(A)}}(t)
$$

These compact relations resting on one-body density operators only [the two-body density operators in (19) are assembled from one-body density operators according to $(5,6)]$ will be used to reformulate $\mathrm{MCTDHF}$ and $\mathrm{MCTDHB}$ in a recursive manner in the following subsection II B.

\section{B. Equations-of-motion utilizing one-body density operators and Combinadic-} based mapping

We can derive (reconstruct) the MCTDHF and MCTDHB equations-of-motion, taking into account a-priori that matrix elements of the form of (15) enter the variational formulation. Within the Lagrangian formulation $[32,69,70]$ of the (Dirac-Frenkel $[71,72]$ ) timedependent variational principle, the action functional of the time-dependent many-particle Schrödinger equation takes on the following form:

$$
\begin{array}{r}
S\left[\left\{C_{J_{A}}(t)\right\},\left\{\phi_{k}(\mathbf{x}, t)\right\}\right]=\int d t\left\{\left\langle\Psi^{(A)}(t)\left|\hat{H}^{(A)}-i \frac{\partial}{\partial t}\right| \Psi^{(A)}(t)\right\rangle-\right. \\
\left.-\sum_{k, j}^{M_{A}} \mu_{k j}^{(A)}(t)\left[\left\langle\phi_{k} \mid \phi_{j}\right\rangle-\delta_{k j}\right]-\varepsilon^{(A)}(t)\left[\sum_{J_{A}=1}^{N_{c o n f}^{(A)}}\left|C_{J_{A}}(t)\right|^{2}-1\right]\right\},
\end{array}
$$

where the time-dependent Lagrange multipliers $\left\{\mu_{k j}^{(A)}(t)\right\}$ are introduced to guarantee the orthonormality of the orbitals at all times. Furthermore, they enable one to first evaluate the expectation value of $\hat{H}^{(A)}-i \frac{\partial}{\partial t}$ with respect to $\left|\Psi^{(A)}(t)\right\rangle$ and then to perform the variation, which is precisely what is needed in order to exploit the Combinadic-based mapping [60] a-priori in the derivation of the equations-of-motion. The (here redundant) time-dependent Lagrange multiplier $\varepsilon^{(A)}(t)$ ensures normalization of the expansion coefficients at all times, and would resurface in the static theory in the case of the imaginary-time-propagation formulation.

To perform the variation of the action functional with respect to the coefficients, we

express the expectation value $\left\langle\Psi^{(A)}(t)\left|\hat{H}^{(A)}-i \frac{\partial}{\partial t}\right| \Psi^{(A)}(t)\right\rangle$ following the Combinadic-based 
mapping [60] and the compact expression in Eq. (15):

$$
\left\langle\Psi^{(A)}(t)\left|\hat{H}^{(A)}-i \frac{\partial}{\partial t}\right| \Psi^{(A)}(t)\right\rangle=\sum_{J_{A}=1}^{N_{c o n f}^{(A)}} C_{J_{A}}^{*}(t)\left[C_{J_{A}}^{\hat{H}^{(A)}-i \frac{\partial}{\partial t}}(A)(t)-i \dot{C}_{J_{A}}(t)\right] .
$$

Representation (22) makes it clear what the variation with respect to the coefficients $\left\{C_{J_{A}}^{*}(t)\right\}$ would lead to. When this variation is performed explicitly, one immediately finds:

$$
C_{J_{A}}^{\hat{H}^{(A)}-i \frac{\partial}{\partial t}^{(A)}}(t)=i \dot{C}_{J_{A}}(t), \quad \forall J_{A} .
$$

The meaning of $i \frac{\partial}{\partial t}^{(A)}$ is that the time-derivative is a one-body operator in the $A$-species Fock (and orbital) space. According to the rules of the previous subsection II A, the left-hand-side of Eq. (23) is given by the sum of its one-, two- and three-body constituents:

$$
C_{J_{A}}^{\hat{H}^{(A)}-i \frac{\partial}{\partial t}^{(A)}}(t)=C_{J_{A}}^{\hat{h}^{(A)}-i \frac{\partial}{\partial t}}{ }^{(A)}(t)+C_{J_{A}}^{\hat{W}^{(A)}}(t)+C_{J_{A}}^{\hat{U}^{(A)}}(t) .
$$

The invariance of $\left|\Psi^{(A)}(t)\right\rangle$ to unitary transformations of the orbitals, compensated by the 'reverse' transformations of the orbitals is well-known [7, 8, 32 and can be represented as follows: $\left|\Psi^{(A)}(t)\right\rangle=\sum_{J_{A}=1}^{N_{\text {conf }}^{(A)}} C_{J_{A}}(t)\left|J_{A} ; t\right\rangle=$ $\sum_{J_{A}=1}^{N_{\text {conf }}^{(A)}} \bar{C}_{J_{A}}(t) \overline{\left|J_{A} ; t\right\rangle}$, with obvious notation. This invariance can be utilized to bring the equations-of-motion into a simpler form (see, in particular, the discussion below on the orbitals' part). Primarily, the differential conditions first introduced by the MCTDH founders $[7,8]$ :

$$
\left\{i \frac{\partial}{\partial t}^{(A)}\right\}_{k q} \equiv i\left\langle\phi_{k} \mid \dot{\phi}_{q}\right\rangle=0, \quad k, q=1, \ldots, M_{A},
$$

come out explicitly from such a unitary transformation [32, 59] and straightforwardly lead in the case of the equations-of-motion for the coefficients to:

$$
\begin{aligned}
& C_{J_{A}}^{\hat{H}^{(A)}}(t)=i \dot{C}_{J_{A}}(t), \quad \forall J_{A}, \\
& C_{J_{A}}^{\hat{H}^{(A)}}(t)=C_{J_{A}}^{\hat{h}^{(A)}}(t)+C_{J_{A}}^{\hat{W}^{(A)}}(t)+C_{J_{A}}^{\hat{U}^{(A)}}(t) .
\end{aligned}
$$

For the general form of the differential conditions, Eq. (25), see the literature [9, 10]. We remark that a particular interesting representation (put forward and utilized so far for distinguishable degrees-of-freedom only) of the differential conditions can be made in order to propagate the systems' natural orbitals $[73,74]$.

In MCTDHF and MCTDHB the integration of the coefficients' part in time is performed (for unitary time-evolution) by the short iterative Lanczos (SIL) algorithm [75]. We remark 
on the numerical implementation of Eq. (26) within SIL propagation [62]. For the SIL one needs to operate with powers of $\hat{H}$ onto the many-particle wave-function and construct the $K$-dimensional Krylov subspace: $\left\{\left|\Psi^{(A)}(t)\right\rangle, \hat{H}^{(A)}\left|\Psi^{(A)}(t)\right\rangle, \ldots, \hat{H}^{(A)}{ }^{K-1}\left|\Psi^{(A)}(t)\right\rangle\right\}$. In the language of the Combinadic-based mapping of coefficients and utilizing the recipe of how to operate with operators on the many-particle wave-function discussed above [60], this construction translates to: $\left\{C_{J_{A}}(t), C_{J_{A}}^{\hat{H}^{(A)}}(t), C_{J_{A}}^{\hat{H}^{(A)} \hat{H}^{(A)}}(t), \ldots\right\}$.

Let us now move to the equations-of-motion for the orbitals $\left\{\phi_{k}(\mathbf{x}, t)\right\}$. For this, the expectation value of the many-body Hamiltonian $\hat{H}^{(A)}$ with respect to $\left|\Psi^{(A)}(t)\right\rangle$ has to be expressed in a form which allows for variation with respect to the orbitals, namely as an explicit function of the quantities (integrals) $h_{k q}^{(A)}, W_{k s q l}^{(A)}$ and $U_{k s p q l r}^{(A)}$ in (4). The result reads:

$$
\begin{aligned}
& \left\langle\Psi\left|\hat{H}^{(A)}-i \frac{\partial}{\partial t}\right| \Psi\right\rangle=\sum_{k, q=1}^{M_{A}} \rho_{k q}^{(A)}\left[h_{k q}^{(A)}-\left\{i \frac{\partial}{\partial t}^{(A)}\right\}_{k q}\right]+ \\
& +\frac{1}{2} \sum_{k, s, l, q=1}^{M_{A}} \rho_{k s l q}^{(A)} W_{k s q l}^{(A)}+\frac{1}{6} \sum_{k, s, p, r, l, q=1}^{M_{A}} \rho_{k s p r l q}^{(A)} U_{k s p q l r}^{(A)}-\sum_{J_{A}=1}^{N_{c o n f}^{(A)}} i C_{J_{A}}^{*}(t) \dot{C}_{J_{A}}(t) .
\end{aligned}
$$

The expectation values of the density operators $\hat{\rho}_{k q}^{(A)}, \hat{\rho}_{k s l q}^{(A)}$ and $\hat{\rho}_{k s p r l q}^{(A)}$ with respect to $\left|\Psi^{(A)}(t)\right\rangle$ (resulting from the expectation value of the Hamiltonian with respect to many-particle wavefunction) are computed following Eq. (15):

$$
\begin{gathered}
\rho_{k q}^{(A)}=\sum_{J_{A}=1}^{N_{c o n f}^{(A)}} C_{J_{A}}^{*}(t) C_{J_{A}}^{\hat{\rho}_{k q}^{(A)}}(t), \quad \rho_{k s l q}^{(A)}=\sum_{J_{A}=1}^{N_{c o n f}^{(A)}} C_{J_{A}}^{*}(t) C_{J_{A}}^{\hat{\rho}_{k s l q}^{(A)}}(t), \\
\rho_{k s p r l q}^{(A)}=\sum_{J_{A}=1}^{N_{c o n f}^{(A)}} C_{J_{A}}^{*}(t) C_{J_{A}}^{\hat{\rho}_{k s p r l q}^{(A)}}(t),
\end{gathered}
$$

where the coefficients $C_{J_{A}}^{\hat{\rho}_{k q}^{(A)}}(t), C_{J_{A}}^{\hat{\rho}_{k s l q}^{(A)}}(t)$ and $C_{J_{A}}^{\hat{\rho}_{k s p r l q}^{(A)}}(t)$ are given in Eqs. $(12,13)$ and $(14)$, respectively. We collect the expectation values of the one-body density operators as the $\operatorname{matrix} \boldsymbol{\rho}^{(A)}(t)=\left\{\rho_{k q}^{(A)}(t)\right\}$.

One should remember that the expectation values of two- and three-body density operators can generally not be factorized into products of expectation values of one-body density operators. For instance (and in the language of the Combinadic-based mapping of coefficients), $C_{J_{A}}^{\hat{\rho}_{k s l q}^{(A)}}(t)= \pm \delta_{s l} C_{J_{A}}^{\hat{\rho}_{k q}^{(A)}}(t) \mp C_{J_{A}}^{\hat{\rho}_{s q}^{(A)} \hat{\rho}_{k l}^{(A)}}(t) \neq \pm \delta_{s l} C_{J_{A}}^{\hat{\rho}_{k q}^{(A)}}(t) \mp C_{J_{A}}^{\hat{\rho}_{k l}^{(A)}}(t) C_{J_{A}}^{\hat{\rho}_{s q}^{(A)}}(t)$. This is unlike the operation of the density operators themselves on the many-particle wave-function utilized above. 
We can now perform the variation of $S\left[\left\{C_{J_{A}}(t)\right\},\left\{\phi_{k}(\mathbf{x}, t)\right\}\right]$ with respect to the orbitals. This variation has been detailed in the literature, see $[32,57]$, and we give here the main steps in the derivation of the equations-of-motion as far as they are needed for our needs later on. Making use of the orthonormality relation between the time-dependent orbitals $\left\{\phi_{k}(\mathbf{x}, t)\right\}$, we can solve for the Lagrange multipliers, $k, j=1, \ldots, M_{A}$ :

$$
\begin{aligned}
& \mu_{k j}^{(A)}(t)= \\
& =\left\langle\phi_{j}\left|\sum_{q=1}^{M_{A}}\left(\rho_{k q}^{(A)}\left[\hat{h}^{(A)}-i \frac{\partial}{\partial t}^{(A)}\right]+\sum_{s, l=1}^{M_{A}} \rho_{k s l q}^{(A)} \hat{W}_{s l}^{(A)}+\frac{1}{2} \sum_{s, p, r, l=1}^{M_{A}} \rho_{k s p r l q}^{(A)} \hat{U}_{s p l r}^{(A)}\right)\right| \phi_{q}\right\rangle .
\end{aligned}
$$

The Lagrange multipliers $\left\{\mu_{k j}^{(A)}(t)\right\}$ can be eliminated from the equations-of-motion which is achieved by the introduction of the projection operator:

$$
\hat{\mathbf{P}}^{(A)}=1-\sum_{u=1}^{M_{A}}\left|\phi_{u}\right\rangle\left\langle\phi_{u}\right| .
$$

When this is done, we find the following equations-of-motion for the orbitals $\left\{\phi_{j}(\mathbf{x}, t)\right\}$, $j=1, \ldots, M_{A}$ :

$$
\begin{aligned}
& \hat{\mathbf{P}}^{(A)} i\left|\dot{\phi}_{j}\right\rangle=\hat{\mathbf{P}}^{(A)}\left[\hat{h}^{(A)}\left|\phi_{j}\right\rangle+\right. \\
& \left.+\sum_{k, q=1}^{M_{A}}\left\{\boldsymbol{\rho}^{(A)}(t)\right\}_{j k}^{-1} \sum_{s, l=1}^{M_{A}}\left(\rho_{k s l q}^{(A)} \hat{W}_{s l}^{(A)}+\frac{1}{2} \sum_{p, r=1}^{M_{A}} \rho_{k s p r l q}^{(A)} \hat{U}_{s p l r}^{(A)}\right)\left|\phi_{q}\right\rangle\right],
\end{aligned}
$$

where

$$
\begin{aligned}
& \hat{W}_{s l}^{(A)}(\mathbf{x}, t)=\int \phi_{s}^{*}\left(\mathbf{x}^{\prime}, t\right) \hat{W}^{(A)}\left(\mathbf{x}, \mathbf{x}^{\prime}\right) \phi_{l}\left(\mathbf{x}^{\prime}, t\right) d \mathbf{x}^{\prime}, \\
& \hat{U}_{s p l r}^{(A)}(\mathbf{x}, t)=\iint \phi_{s}^{*}\left(\mathbf{x}^{\prime}, t\right) \phi_{p}^{*}\left(\mathbf{x}^{\prime \prime}, t\right) \hat{U}^{(A)}\left(\mathbf{x}, \mathbf{x}^{\prime}, \mathbf{x}^{\prime \prime}\right) \phi_{l}\left(\mathbf{x}^{\prime}, t\right) \phi_{r}\left(\mathbf{x}^{\prime \prime}, t\right) d \mathbf{x}^{\prime} d \mathbf{x}^{\prime \prime},
\end{aligned}
$$

are local (for spin-independent interactions), time-dependent one-body potentials, and $\dot{\phi}_{j} \equiv$ $\frac{\partial \phi_{j}}{\partial t}$.

Utilizing the differential conditions $(25)$ we can eliminate the projection operator $\hat{\mathbf{P}}^{(A)}$ appearing on the left-hand-side of Eq. (31) and arrive at the final result for the equationsof-motion of the orbitals in MCTDHF and MCTDHB (see $[10,57]), j=1, \ldots, M_{A}$ :

$$
\begin{aligned}
& i\left|\dot{\phi}_{j}\right\rangle=\hat{\mathbf{P}}^{(A)}\left[\hat{h}^{(A)}\left|\phi_{j}\right\rangle+\right. \\
& \left.+\sum_{k, q=1}^{M_{A}}\left\{\boldsymbol{\rho}^{(A)}(t)\right\}_{j k}^{-1} \sum_{s, l=1}^{M_{A}}\left(\rho_{k s l q}^{(A)} \hat{W}_{s l}^{(A)}+\frac{1}{2} \sum_{p, r=1}^{M_{A}} \rho_{k s p r l q}^{(A)} \hat{U}_{s p l r}^{(A)}\right)\left|\phi_{q}\right\rangle\right] .
\end{aligned}
$$


Summarizing, the coupled sets of equations-of-motion (26) for the expansion coefficients and (33) for the orbitals constitute the MCTDHF and MCTDHB methods, where the one-body density operators $(5,6)$ are employed as the basic building bricks in their construction and implementation.

We can also propagate the MCTDHF and MCTDHB equations-of-motion $(26,33)$ in imaginary time and arrive for time-independent Hamiltonians at the corresponding selfconsistent static theories, MCHF [76, 77] and MCHB [56]. Thus, setting $t \rightarrow-i t$ into the coupled sets $(23,31)$ or into $(26,33)$, and translating back from the projection operator $\hat{\mathbf{P}}^{(A)}$ to the Lagrange multipliers $\left\{\mu_{k j}^{(A)}\right\}$, the final result reads, $k=1, \ldots, M_{A}$ :

$$
\begin{gathered}
\sum_{q=1}^{M_{A}}\left[\rho_{k q}^{(A)} \hat{h}^{(A)}+\sum_{s, l=1}^{M_{A}}\left(\rho_{k s l q}^{(A)} \hat{W}_{s l}^{(A)}+\frac{1}{2} \sum_{p, r=1}^{M_{A}} \rho_{k s p r l q}^{(A)} \hat{U}_{s p l r}^{(A)}\right)\right]\left|\phi_{q}\right\rangle=\sum_{j=1}^{M_{A}} \mu_{k j}^{(A)}\left|\phi_{j}\right\rangle \\
C_{J_{A}}^{\hat{H}^{(A)}}=\varepsilon^{(A)} C_{J_{A}}, \quad \forall J_{A}
\end{gathered}
$$

where, making use of the normalization of the many-particle wave-function, $\varepsilon^{(A)}=$ $\sum_{J_{A}=1}^{N_{\text {conf }}^{(A)}} C_{J_{A}}^{*} C_{J_{A}}^{\hat{H}^{(A)}}$ is the eigen-energy of the system. Making use of the fact that the matrix of Lagrange multipliers $\left\{\mu_{k j}^{(A)}\right\}$ is Hermitian (for stationary states) and of the invariance property of the multiconfigurational wave-function (to unitary transformations of the orbitals compensated by the 'reverse' transformations of the coefficients), one can transform Eq. (34) to a representation where $\left\{\mu_{k j}^{(A)}\right\}$ is a diagonal matrix.

All in all, we have formulated in the present section the MCTDHF and MCTDHB equations-of-motion, as well as their static variants MCHF and MCHB, by (i) utilizing in a recursive manner one-body density operators only, and by (ii) employing a priori the Combinadic-based mapping formulation of Ref. [60] to evaluate matrix elements. This sets up the tools to put forward the MCTDH theory for mixtures of three kinds of identical particles in the following Sec. III, and to briefly discuss its structure and properties, and how to implement it.

\section{THREE KINDS OF IDENTICAL PARTICLES: MCTDH-FFF, MCTDH-BFF, MCTDH-BBF AND MCTDH-BBB}

In the present section we specify the MCTDH theory for mixtures of three kinds of identical particles, interacting with up to three-body forces. Before we get into the details of 
derivation and flood of equations, we would like to lay out a general scheme or flowchart that one can follow to handle similar or even more complex mixtures. Specifically, we need to assign a different set of time-dependent orthonormal orbitals to each and every species in the mixture. These orbitals are then used to assemble the time-dependent configurations (with determinants' parts for fermions and permanents' parts for bosons). The manyparticle wave-function is thereafter assembled as a linear combination of all time-dependent configurations with time-dependent expansion coefficients. The many-particle Hamiltonian contains different terms: It contains intra-species terms and inter-species terms which consist of two-body, three-body and so on interactions. The main point in the representation of the Hamiltonian is the utilization of one-body density operators. In turn, all intra-species and inter-species interactions can be represented utilizing (products of) one-body density operators only.

The key step in the derivation of the equations-of-motion is the utilization of the Lagrangian formulation $[32,69,70]$ of the (Dirac-Frenkel [71, 72]) time-dependent variational principle with Lagrange multipliers for each species' orbitals, ensuring thereby the orthonormality of the orbitals for all times. In such a way, matrix-elements appear within the formulation explicitly, before the variation with respect to either the expansion coefficients or the orbitals is performed. The equations-of-motion for the expansion coefficients of the multiconfigurational wave-function are obtained by taking the variation of the action functional when it is expressed explicitly in terms of the expansion coefficients. The Combinadic-based mapping [60] lifts the necessity to work with the huge matrix representation of the Hamiltonian with respect to the configurations, and allows one to efficiently perform operations on the vector of expansion coefficients directly. The equations-of-motion for the orbitals are obtained by taking the variation of the action functional when it is expressed explicitly in terms of the (integrals of the) orbitals. When this is performed, expectation values of the various density operators in the Hamiltonian (with respect to the many-particle wavefunction) emerge which can be efficiently computed utilizing the Combinadic-based mapping [60]. 


\section{A. Additional ingredients for mixtures}

For a mixture of three kinds of identical particles, $N_{A}$ particles of type $A, N_{B}$ particles of type $B$ and $N_{C}$ particles of type $C$, we need now two additional field operators expanded by different complete sets of time-dependent orbitals:

$$
\hat{\mathbf{\Psi}}_{B}(\mathbf{y})=\sum_{k^{\prime}} \hat{b}_{k^{\prime}}(t) \psi_{k^{\prime}}(\mathbf{y}, t), \quad \hat{\Psi}_{C}(\mathbf{z})=\sum_{k^{\prime \prime}} \hat{c}_{k^{\prime \prime}}(t) \chi_{k^{\prime \prime}}(\mathbf{z}, t),
$$

where the field operator for the $A$-species particles $\hat{\mathbf{\Psi}}_{A}(\mathbf{x})$ was first introduced and expanded in (2). Note that each species can have a different spin, hence the explicit three distinct coordinates $\mathbf{x}, \mathbf{y}$ and $\mathbf{z}$. Field operators of distinct particles (can be chosen to) commute.

Our starting point is the many-body Hamiltonian of the most general 3-species mixture with up to 3-body interactions:

$$
\begin{aligned}
& \hat{H}^{(A B C)}=\hat{H}^{(A)}+\hat{H}^{(B)}+\hat{H}^{(C)}+\hat{W}^{(A B)}+\hat{W}^{(A C)}+\hat{W}^{(B C)}+ \\
& +\hat{U}^{(A A B)}+\hat{U}^{(A B B)}+\hat{U}^{(A A C)}+\hat{U}^{(A C C)}+\hat{U}^{(B B C)}+\hat{U}^{(B C C)}+\hat{U}^{(A B C)} .
\end{aligned}
$$

Here, $\hat{H}^{(A)}, \hat{H}^{(B)}$ and $\hat{H}^{(C)}$ are the single-species Hamiltonians that can be read of (1). The inter-species two-body interaction parts are given by:

$$
\begin{aligned}
\hat{W}^{(A B)} & =\iint d \mathbf{x} d \mathbf{y} \hat{\mathbf{\Psi}}_{A}^{\dagger}(\mathbf{x}) \hat{\mathbf{\Psi}}_{B}^{\dagger}(\mathbf{y}) \hat{W}^{(A B)}(\mathbf{x}, \mathbf{y}) \hat{\mathbf{\Psi}}_{B}(\mathbf{y}) \hat{\mathbf{\Psi}}_{A}(\mathbf{x}), \\
\hat{W}^{(A C)} & =\iint d \mathbf{x} d \mathbf{z} \hat{\mathbf{\Psi}}_{A}^{\dagger}(\mathbf{x}) \hat{\mathbf{\Psi}}_{C}^{\dagger}(\mathbf{z}) \hat{W}^{(A C)}(\mathbf{x}, \mathbf{z}) \hat{\mathbf{\Psi}}_{C}(\mathbf{z}) \hat{\mathbf{\Psi}}_{A}(\mathbf{x}), \\
\hat{W}^{(B C)} & =\iint d \mathbf{y} d \mathbf{z} \hat{\mathbf{\Psi}}_{B}^{\dagger}(\mathbf{y}) \hat{\mathbf{\Psi}}_{C}^{\dagger}(\mathbf{z}) \hat{W}^{(B C)}(\mathbf{y}, \mathbf{z}) \hat{\mathbf{\Psi}}_{C}(\mathbf{z}) \hat{\mathbf{\Psi}}_{B}(\mathbf{y}) .
\end{aligned}
$$

The inter-species three-body interaction parts, resulting from the force between two identical 
particles and a third distinct particle, are given by:

$$
\begin{aligned}
& \hat{U}^{(A A B)}=\frac{1}{2} \iiint d \mathbf{x} d \mathbf{x}^{\prime} d \mathbf{y} \hat{\Psi}_{A}^{\dagger}(\mathbf{x}) \hat{\mathbf{\Psi}}_{A}^{\dagger}\left(\mathbf{x}^{\prime}\right) \hat{\mathbf{\Psi}}_{B}^{\dagger}(\mathbf{y}) \hat{U}^{(A A B)}\left(\mathbf{x}, \mathbf{x}^{\prime}, \mathbf{y}\right) \times \\
& \times \hat{\boldsymbol{\Psi}}_{B}(\mathbf{y}) \hat{\boldsymbol{\Psi}}_{A}\left(\mathbf{x}^{\prime}\right) \hat{\boldsymbol{\Psi}}_{A}(\mathbf{x}), \\
& \hat{U}^{(A B B)}=\frac{1}{2} \iiint d \mathbf{x} d \mathbf{y} d \mathbf{y}^{\prime} \hat{\Psi}_{A}^{\dagger}(\mathbf{x}) \hat{\mathbf{\Psi}}_{B}^{\dagger}(\mathbf{y}) \hat{\mathbf{\Psi}}_{B}^{\dagger}\left(\mathbf{y}^{\prime}\right) \hat{U}^{(A B B)}\left(\mathbf{x}, \mathbf{y}, \mathbf{y}^{\prime}\right) \times \\
& \times \hat{\boldsymbol{\Psi}}_{B}\left(\mathbf{y}^{\prime}\right) \hat{\boldsymbol{\Psi}}_{B}(\mathbf{y}) \hat{\boldsymbol{\Psi}}_{A}(\mathbf{x}), \\
& \hat{U}^{(A A C)}=\frac{1}{2} \iiint d \mathbf{x} d \mathbf{x}^{\prime} d \mathbf{z} \hat{\mathbf{\Psi}}_{A}^{\dagger}(\mathbf{x}) \hat{\mathbf{\Psi}}_{A}^{\dagger}\left(\mathbf{x}^{\prime}\right) \hat{\mathbf{\Psi}}_{C}^{\dagger}(\mathbf{z}) \hat{U}^{(A A C)}\left(\mathbf{x}, \mathbf{x}^{\prime}, \mathbf{z}\right) \times \\
& \times \hat{\mathbf{\Psi}}_{C}(\mathbf{z}) \hat{\mathbf{\Psi}}_{A}\left(\mathbf{x}^{\prime}\right) \hat{\mathbf{\Psi}}_{A}(\mathbf{x}), \\
& \hat{U}^{(A C C)}=\frac{1}{2} \iiint d \mathbf{x} d \mathbf{z} d \mathbf{z}^{\prime} \hat{\mathbf{\Psi}}_{A}^{\dagger}(\mathbf{x}) \hat{\mathbf{\Psi}}_{C}^{\dagger}(\mathbf{z}) \hat{\mathbf{\Psi}}_{C}^{\dagger}\left(\mathbf{z}^{\prime}\right) \hat{U}^{(A C C)}\left(\mathbf{x}, \mathbf{z}, \mathbf{z}^{\prime}\right) \times \\
& \times \hat{\mathbf{\Psi}}_{C}\left(\mathbf{z}^{\prime}\right) \hat{\mathbf{\Psi}}_{C}(\mathbf{z}) \hat{\mathbf{\Psi}}_{A}(\mathbf{x}), \\
& \hat{U}^{(B B C)}=\frac{1}{2} \iiint d \mathbf{y} d \mathbf{y}^{\prime} d \mathbf{z} \hat{\mathbf{\Psi}}_{B}^{\dagger}(\mathbf{y}) \hat{\mathbf{\Psi}}_{B}^{\dagger}\left(\mathbf{y}^{\prime}\right) \hat{\mathbf{\Psi}}_{C}^{\dagger}(\mathbf{z}) \hat{U}^{(B B C)}\left(\mathbf{y}, \mathbf{y}^{\prime}, \mathbf{z}\right) \times \\
& \times \hat{\mathbf{\Psi}}_{C}(\mathbf{z}) \hat{\mathbf{\Psi}}_{B}\left(\mathbf{y}^{\prime}\right) \hat{\mathbf{\Psi}}_{B}(\mathbf{y}), \\
& \hat{U}^{(B C C)}=\frac{1}{2} \iiint d \mathbf{y} d \mathbf{z} d \mathbf{z}^{\prime} \hat{\mathbf{\Psi}}_{B}^{\dagger}(\mathbf{y}) \hat{\mathbf{\Psi}}_{C}^{\dagger}(\mathbf{z}) \hat{\mathbf{\Psi}}_{C}^{\dagger}\left(\mathbf{z}^{\prime}\right) \hat{U}^{(B C C)}\left(\mathbf{y}, \mathbf{z}, \mathbf{z}^{\prime}\right) \times \\
& \times \hat{\Psi}_{C}\left(\mathbf{z}^{\prime}\right) \hat{\Psi}_{C}(\mathbf{z}) \hat{\Psi}_{B}(\mathbf{y}) \text {. }
\end{aligned}
$$

Finally, the inter-species three-body interaction part, resulting from the force between three different particles is given by:

$$
\begin{aligned}
\hat{U}^{(A B C)}= & \iiint d \mathbf{x} d \mathbf{y} d \mathbf{z} \hat{\mathbf{\Psi}}_{A}^{\dagger}(\mathbf{x}) \hat{\mathbf{\Psi}}_{B}^{\dagger}(\mathbf{y}) \hat{\mathbf{\Psi}}_{C}^{\dagger}(\mathbf{z}) \hat{U}^{(A B C)}(\mathbf{x}, \mathbf{y}, \mathbf{z}) \times \\
& \times \hat{\mathbf{\Psi}}_{C}(\mathbf{z}) \hat{\mathbf{\Psi}}_{B}(\mathbf{y}) \hat{\mathbf{\Psi}}_{A}(\mathbf{x}) .
\end{aligned}
$$

When all the above are combined, i.e., the field operators $\hat{\mathbf{\Psi}}_{A}(\mathbf{x}), \hat{\mathbf{\Psi}}_{B}(\mathbf{y})$ and $\hat{\mathbf{\Psi}}_{B}(\mathbf{z})$ substituted into the various interaction terms, we find the following second-quantized expression 
for the mixture's Hamiltonian:

$$
\begin{aligned}
& \hat{H}^{(A B C)}=\sum_{k, q} h_{k q}^{(A)} \hat{\rho}_{k q}^{(A)}+\frac{1}{2} \sum_{k, s, q, l} W_{k s q l}^{(A)} \hat{\rho}_{k s l q}^{(A)}+\frac{1}{6} \sum_{k, s, p, r, l, q} U_{k s p q l r}^{(A)} \hat{\rho}_{k s p r l q}^{(A)}+ \\
& +\sum_{k^{\prime}, q^{\prime}} h_{k^{\prime} q^{\prime}}^{(B)} \hat{\rho}_{k^{\prime} q^{\prime}}^{(B)}+\frac{1}{2} \sum_{k^{\prime}, s^{\prime}, q^{\prime}, l^{\prime}} W_{k^{\prime} s^{\prime} q^{\prime} l^{\prime}}^{(B)} \hat{\rho}_{k^{\prime} s^{\prime} l^{\prime} q^{\prime}}^{(B)}+\frac{1}{6} \sum_{k^{\prime}, s^{\prime}, p^{\prime}, r^{\prime}, l^{\prime}, q^{\prime}} U_{k^{\prime} s^{\prime} p^{\prime} q^{\prime} l^{\prime} r^{\prime}}^{(B)} \hat{\rho}_{k^{\prime} s^{\prime} p^{\prime} r^{\prime} l^{\prime} q^{\prime}}^{(B)}+ \\
& +\sum_{k^{\prime \prime}, q^{\prime \prime}} h_{k^{\prime \prime} q^{\prime \prime}}^{(C)} \hat{\rho}_{k^{\prime \prime} q^{\prime \prime}}^{(C)}+\frac{1}{2} \sum_{k^{\prime \prime}, s^{\prime \prime}, q^{\prime \prime}, l^{\prime \prime}} W_{k^{\prime \prime} s^{\prime \prime} q^{\prime \prime} l^{\prime \prime}}^{(C)} \hat{\rho}_{k^{\prime \prime} s^{\prime \prime} l^{\prime \prime} q^{\prime \prime}}^{(C)}+ \\
& +\frac{1}{6} \sum_{k^{\prime \prime}, s^{\prime \prime}, p^{\prime \prime}, r^{\prime \prime}, l^{\prime \prime}, q^{\prime \prime}} U_{k^{\prime \prime} s^{\prime \prime} p^{\prime \prime} q^{\prime \prime} l^{\prime \prime} r^{\prime \prime}}^{(C)} \hat{\rho}_{k^{\prime \prime} s^{\prime \prime} p^{\prime \prime} r^{\prime \prime} l^{\prime \prime} q^{\prime \prime}}^{(C)}+ \\
& +\sum_{k, k^{\prime}, q, q^{\prime}} W_{k k^{\prime} q q^{\prime}}^{(A B)} \hat{\rho}_{k q}^{(A)} \hat{\rho}_{k^{\prime} q^{\prime}}^{(B)}+\sum_{k, k^{\prime \prime}, q, q^{\prime \prime}} W_{k k^{\prime \prime} q q^{\prime \prime}}^{(A C)} \hat{\rho}_{k q}^{(A)} \hat{\rho}_{k^{\prime \prime} q^{\prime \prime}}^{(C)}+\sum_{k^{\prime}, k^{\prime \prime}, q^{\prime}, q^{\prime \prime}} W_{k^{\prime} k^{\prime \prime} q^{\prime} q^{\prime \prime}}^{(B C)} \hat{\rho}_{k^{\prime} q^{\prime}}^{(B)} \hat{\rho}_{k^{\prime \prime} q^{\prime \prime}}^{(C)}+ \\
& +\frac{1}{2} \sum_{k, k^{\prime}, s, q, q^{\prime}, l} U_{k k^{\prime} s q q^{\prime} l}^{(A A B)} \hat{\rho}_{k s l q}^{(A)} \hat{\rho}_{k^{\prime} q^{\prime}}^{(B)}+\frac{1}{2} \sum_{k, k^{\prime}, s^{\prime}, q, q^{\prime}, l^{\prime}} U_{k k^{\prime} s^{\prime} q q^{\prime} l^{\prime}}^{(A B B)} \hat{\rho}_{k q}^{(A)} \hat{\rho}_{k^{\prime} s^{\prime} l^{\prime} q^{\prime}}^{(B)}+ \\
& +\frac{1}{2} \sum_{k, k^{\prime \prime}, s, q, q^{\prime \prime}, l} U_{k k^{\prime \prime} s q q^{\prime \prime l} l}^{(A A C)} \hat{\rho}_{k s l q}^{(A)} \hat{\rho}_{k^{\prime \prime} q^{\prime \prime}}^{(C)}+\frac{1}{2} \sum_{k, k^{\prime \prime}, s^{\prime \prime}, q, q^{\prime \prime}, l^{\prime \prime}} U_{k k^{\prime \prime} s^{\prime \prime} q q^{\prime \prime} l^{\prime \prime}}^{(A C C)} \hat{\rho}_{k q}^{(A)} \hat{\rho}_{k^{\prime \prime} s^{\prime \prime} l^{\prime \prime} q^{\prime \prime}}^{(C)}+ \\
& +\frac{1}{2} \sum_{k^{\prime}, k^{\prime \prime}, s^{\prime}, q^{\prime}, q^{\prime \prime}, l^{\prime}} U_{k^{\prime} k^{\prime \prime} s^{\prime} q^{\prime} q^{\prime \prime} l^{\prime}}^{(B B C)} \hat{\rho}_{k^{\prime} s^{\prime} l^{\prime} q^{\prime}}^{(B)} \hat{\rho}_{k^{\prime \prime} q^{\prime \prime}}^{(C)}+\frac{1}{2} \sum_{k^{\prime}, k^{\prime \prime}, s^{\prime \prime}, q^{\prime}, q^{\prime \prime}, l^{\prime \prime}} U_{k^{\prime} k^{\prime \prime} s^{\prime \prime} q^{\prime} q^{\prime \prime} l^{\prime \prime}}^{(B C C)} \hat{\rho}_{k^{\prime} q^{\prime}}^{(B)} \hat{\rho}_{k^{\prime \prime} s^{\prime \prime} l^{\prime \prime} q^{\prime \prime}}^{(C)}+ \\
& +\sum_{k, k^{\prime}, k^{\prime \prime}, q, q^{\prime}, q^{\prime \prime}} U_{k k^{\prime} k^{\prime \prime} q q^{\prime} q^{\prime \prime}}^{(A B C)} \hat{\rho}_{k q}^{(A)} \hat{\rho}_{k^{\prime} q^{\prime}}^{(B)} \hat{\rho}_{k^{\prime \prime} q^{\prime \prime}}^{(C)}
\end{aligned}
$$

$\hat{H}^{(A B C)}$ governs the non-equilibrium dynamics (and statics) of the mixture, and the most efficient way to treat this dynamics is by specifying the MCTDH method for the mixture, making use of the building bricks of the previous section II. We see in (40) two kinds of ingredients. First, there are matrix elements (integrals) of the various interaction terms with respect to the orbitals. For the flow of exposition and for completeness, we list them in Appendix C. Second, there are various density operators in $\hat{H}^{(A B C)}$. The $B$ and $C$ intra-species density operators can be read directly from Eqs. (5,6), when replacing therein the $A$-species quantities. The inter-species density operators in (40) can all be represented as appropriate products of the one-body density operators: $\left\{\hat{\rho}_{k q}^{(A)}\right\},\left\{\hat{\rho}_{k^{\prime} q^{\prime}}^{(B)}\right\}$ and $\left\{\hat{\rho}_{k^{\prime \prime} q^{\prime \prime}}^{(C)}\right\}$. These are the (basic) building bricks of our theory for mixtures. But how to operate with them on many-particle wave-functions of mixtures?

The multiconfigurational ansatz for a mixture of three kinds of identical particles now takes on the from:

$$
\left|\Psi^{(A B C)}(t)\right\rangle=\sum_{J_{A}=1}^{N_{\text {conf }}^{(A)}} \sum_{J_{B}=1}^{N_{\text {conf }}^{(B)}} \sum_{J_{C}=1}^{N_{\text {conf }}^{(C)}} C_{J_{A}, J_{B}, J_{C}}(t)\left|J_{A}, J_{B}, J_{C} ; t\right\rangle,
$$


where we denote hereafter $\vec{J}=\left(J_{A}, J_{B}, J_{C}\right)$ for brevity, such that $C_{\vec{J}}(t) \equiv C_{J_{A}, J_{B}, J_{C}}(t)$, $|\vec{J} ; t\rangle \equiv\left|J_{A}, J_{B}, J_{C} ; t\right\rangle$ and $\sum_{\{\vec{J}\}} \equiv \sum_{J_{A}=1}^{N_{c o n f}^{(A)}} \sum_{J_{B}=1}^{N_{c o n f}^{(B)}} \sum_{J_{C}=1}^{N_{c o n f}^{(C)}}$.

To prescribe the action of operators on the multiconfigurational wave-function of the mixture (41), all we need to know is how the density operators operate on $\left|\Psi^{(A B C)}(t)\right\rangle$. The operation of the basic, one-body density operators, whether $\hat{\rho}_{k q}^{(A)}, \hat{\rho}_{k^{\prime} q^{\prime}}^{(B)}$ or $\hat{\rho}_{k^{\prime \prime} q^{\prime \prime}}^{(C)}$ can be read of directly from Eqs. (11-19) and we will not repeat them here (one needs just to replace therein $J_{A}$ by $\vec{J}$ in the overall notation, and $M_{A}$ by $M_{B}$ or $M_{C}$, when appropriate; also see [60]). For the inter-species two-body density operators we have:

$$
C_{\vec{J}}^{\hat{\rho}_{k q}^{(A)} \hat{\rho}_{k^{\prime} q^{\prime}}^{(B)}}(t), \quad C_{\vec{J}}^{\hat{\rho}_{k q}^{(A)} \hat{\rho}_{k^{\prime \prime} q^{\prime \prime}}^{(C)}}(t), \quad C_{\vec{J}}^{\hat{\rho}_{k^{\prime} q^{\prime}}^{(B)} \hat{\rho}_{k^{\prime \prime} q^{\prime \prime}}^{(C)}(t) .}
$$

The notation in (42) is to be understood as follows: The two one-body density operators (in each case) are written as superscripts on the same level, signifying that they commute one with the other; The operation of the two one-body density operators on $\left|\Psi^{(A B C)}(t)\right\rangle$ is to be performed sequentially, i.e., the first operates on $\left|\Psi^{(A B C)}(t)\right\rangle$ and the second operates on the outcome. Finally, for the inter-species three-body density operators we have:

$$
\begin{array}{lcl}
C_{\vec{J}}^{\hat{\rho}_{k s l}^{(A)} \hat{\rho}_{k^{\prime} q^{\prime}}^{(B)}}(t), & C_{\vec{J}}^{\hat{\rho}_{k q}^{(A)} \hat{\rho}_{k^{\prime} s^{\prime} l^{\prime} q^{\prime}}^{(B)}(t),} & C_{\vec{J}}^{\hat{\rho}_{k s l q}^{(A)} \hat{\rho}_{k^{\prime \prime} q^{\prime \prime}}^{(C)}(t),} \\
C_{\vec{J}}^{\hat{\rho}_{k q}^{(A)} \hat{\rho}_{k^{\prime \prime} s^{\prime \prime} l^{\prime \prime} q^{\prime \prime}}^{(C)}}(t), & C_{\vec{J}}^{\hat{\rho}_{k^{\prime} s^{\prime} l^{\prime} q^{\prime}}^{(B)} \hat{\rho}_{k^{\prime \prime} q^{\prime \prime}}^{(C)}}(t), & C_{\vec{J}}^{\hat{\rho}_{k^{\prime} q^{\prime}}^{(B)} \hat{\rho}_{k^{\prime \prime} s^{\prime \prime} l^{\prime \prime} q^{\prime \prime}}^{(C)}(t),}
\end{array}
$$

where the operation of the two-body density operators appearing in the superscripts is further decomposed to operations of one-body density operators on $\left|\Psi^{(A B C)}(t)\right\rangle$ analogously to Eq. (14) [see Appendix A], and

$$
C_{\vec{J}}^{\hat{\rho}_{k q}^{(A)} \hat{\rho}_{k^{\prime} q^{\prime}}^{(B)} \hat{\rho}_{k^{\prime \prime} q^{\prime \prime}}^{(C)}}(t)
$$

Now we are in the position to write the action of operators on the multiconfigurational wave-function of the mixture (41). This is collected for ease of reading and for completeness in Appendix A.

We have gathered most ingredients for the derivation of the equations-of-motion, which is written down in the subsequence section III B. There are four possible mixtures (FermiFermi-Fermi, Bose-Fermi-Fermi, Bose-Bose-Fermi and Bose-Bose-Bose), and the resulting MCTDH-FFF, MCTDH-BFF, MCTDH-BBF and MCTDH-BBB are to be derived and presented in a unified manner, in the spirit it has been done in the single-species case $[10,57]$ (and the previous section II) and for mixtures of two kinds of identical particles [10, 58]. 


\section{B. Equations-of-motion utilizing one-body density operators and Combinadic- based mapping for mixtures}

The action functional of the time-dependent many-particle Schrödinger equation takes on the form:

$$
\begin{aligned}
& S\left[\left\{C_{\vec{J}}(t)\right\},\left\{\phi_{k}(\mathbf{x}, t)\right\},\left\{\psi_{k^{\prime}}(\mathbf{y}, t)\right\},\left\{\chi_{k^{\prime \prime}}(\mathbf{z}, t)\right\}\right]= \\
& \int d t\left\{\left\langle\Psi^{(A B C)}(t)\left|\hat{H}^{(A B C)}-i \frac{\partial}{\partial t}\right| \Psi^{(A B C)}(t)\right\rangle-\right. \\
& -\sum_{k, j}^{M_{A}} \mu_{k j}^{(A)}(t)\left[\left\langle\phi_{k} \mid \phi_{j}\right\rangle-\delta_{k j}\right]-\sum_{k^{\prime}, j^{\prime}}^{M_{B}} \mu_{k^{\prime} j^{\prime}}^{(B)}(t)\left[\left\langle\psi_{k^{\prime}} \mid \psi_{j^{\prime}}\right\rangle-\delta_{k^{\prime} j^{\prime}}\right]- \\
& \left.-\sum_{k^{\prime \prime}, j^{\prime \prime}}^{M_{C}} \mu_{k^{\prime \prime} j^{\prime \prime}}^{(C)}(t)\left[\left\langle\chi_{k^{\prime \prime}} \mid \chi_{j^{\prime \prime}}\right\rangle-\delta_{k^{\prime \prime} j^{\prime \prime}}\right]-\varepsilon^{(A B C)}(t)\left[\sum_{\{\vec{J}\}}\left|C_{\vec{J}}(t)\right|^{2}-1\right]\right\},
\end{aligned}
$$

where the time-dependent Lagrange multipliers $\left\{\mu_{k j}^{(A)}(t)\right\}, \quad\left\{\mu_{k^{\prime} j^{\prime}}^{(B)}(t)\right\} \quad$ and $\left\{\mu_{k^{\prime \prime} j^{\prime \prime}}^{(C)}(t)\right\}$ are introduced, respectively, to ensure the orthonormality of the $A-, B$ and $C$-species orbitals at all times. Note that orbitals of distinct particles need not be orthogonal to each other. As for the single-species theory, the Lagrange multiplier $\varepsilon^{(A B C)}(t)$ is redundant in the time-dependent case and will resurface in the static theory.

In what follows we present the main steps of the derivation. More details and various quantities needed for the derivation and in particular for the implementation of the equations-of-motion are deferred to Appendix B and Appendix C.

To perform the variation of the action functional (45) with respect to the coefficients, we write the expectation value of $\hat{H}^{(A B C)}$ with respect to $\left|\Psi^{(A B C)}(t)\right\rangle$ in a form which is explicit with respect to the coefficients:

$$
\begin{aligned}
& \left\langle\Psi^{(A B C)}(t)\left|\hat{H}^{(A B C)}-i \frac{\partial}{\partial t}\right| \Psi^{(A B C)}(t)\right\rangle= \\
& =\sum_{\{\vec{J}\}} C_{\vec{J}}^{*}(t)\left[C_{\vec{J}}^{\left.\hat{H}^{(A B C)}-i \frac{\partial}{\partial t}{ }^{(A)}-i \frac{\partial}{\partial t}{ }^{(B)}-i \frac{\partial}{\partial t}^{(C)}(t)-i \dot{C}_{\vec{J}}(t)\right] .}\right.
\end{aligned}
$$

The three time-derivative operators $i \frac{\partial}{\partial t}^{(A)}, i \frac{\partial}{\partial t}^{(B)}$ and $i \frac{\partial}{\partial t}^{(C)}$ make it clear that to each species there is associated a different one-body operator representing the derivative of orbitals in time.

Performing the variation of $S\left[\left\{C_{\vec{J}}(t)\right\},\left\{\phi_{k}(\mathbf{x}, t)\right\},\left\{\psi_{k^{\prime}}(\mathbf{y}, t)\right\},\left\{\chi_{k^{\prime \prime}}(\mathbf{z}, t)\right\}\right]$ with respect to the expansion coefficients $\left\{C_{\vec{J}}^{*}(t)\right\}$, we then make use of the differential conditions for 
the orbitals of each species,

$$
\begin{aligned}
& \left\{i \frac{\partial}{\partial t}^{(B)}\right\}_{k^{\prime} q^{\prime}} \equiv i\left\langle\psi_{k^{\prime}} \mid \dot{\psi}_{q^{\prime}}\right\rangle=0, \quad k^{\prime}, q^{\prime}=1, \ldots, M_{B}, \\
& \left\{i \frac{\partial}{\partial t}^{(C)}\right\}_{k^{\prime \prime} q^{\prime \prime}} \equiv i\left\langle\chi_{k^{\prime \prime}} \mid \dot{\chi}_{q^{\prime \prime}}\right\rangle=0, \quad k^{\prime \prime}, q^{\prime \prime}=1, \ldots, M_{C},
\end{aligned}
$$

where the differential conditions with respect to the $A$-spices orbitals have been introduced in (25). This leads to the final result for the equations-of-motion for the expansion coefficients:

$$
\begin{aligned}
& C_{\vec{J}}^{\hat{H}^{(A B C)}}(t)=i \dot{C}_{\vec{J}}(t), \quad \forall \vec{J}, \\
& C_{\vec{J}}^{\hat{H}^{(A B C)}}(t)=C_{\vec{J}}^{\hat{H}^{(A)}}(t)+C_{\vec{J}}^{\hat{H}^{(B)}}(t)+C_{\vec{J}}^{\hat{H}^{(C)}}(t)+ \\
& +C_{\vec{J}}^{\hat{W}^{(A B)}}(t)+C_{\vec{J}}^{\hat{W}^{(A C)}}(t)+C_{\vec{J}}^{\hat{W}^{(B C)}}(t)+ \\
& +C_{\vec{J}}^{\hat{U}^{(A A B)}}(t)+C_{\vec{J}}^{\hat{U}^{(A B B)}}(t)+C_{\vec{J}}^{\hat{U}^{(A A C)}}(t)+C_{\vec{J}}^{\hat{U}^{(A C C)}}(t)+ \\
& +C_{\vec{J}}^{\hat{U}^{(B B C)}}(t)+C_{\vec{J}}^{\hat{U}^{(B C C)}}(t)+C_{\vec{J}}^{\hat{U}^{(A B C)}}(t) .
\end{aligned}
$$

We remark that other forms of the differential conditions $(25,47)$ can be used, in particular, each species can have a different form depending on the physical problem at hand and on numerical needs.

Let us move to the equations-of-motion for the orbitals $\left\{\phi_{k}(\mathbf{x}, t)\right\}$, $\left\{\psi_{k^{\prime}}(\mathbf{y}, t)\right\}$ and $\left\{\chi_{k^{\prime \prime}}(\mathbf{z}, t)\right\}$. For this, we express the expectation value $\left\langle\Psi^{(A B C)}\left|\hat{H}^{(A B C)}-i \frac{\partial}{\partial t}\right| \Psi^{(A B C)}\right\rangle$ in a form which explicitly depends on the various integrals with respect to the orbitals. The result is lengthly and posted in Appendix C. In particular, the expectation values of the various density operators in $\hat{H}^{(A B C)}$ [Eq. (40)] emerge as matrix elements of the different intra-species and inter-species reduced density matrices. For ease of reading and for completeness, we collect in Appendix B all reduced density matrices and their respective matrix elements needed in the theory and its numerical implementation.

We can now proceed and perform the variation of the action functional (45) with respect to the orbitals. Performing the variation with respect to $\left\{\phi_{k}^{*}(\mathbf{x}, t)\right\},\left\{\psi_{k^{\prime}}^{*}(\mathbf{y}, t)\right\}$ and $\left\{\chi_{k^{\prime \prime}}^{*}(\mathbf{z}, t)\right\}$, making use of the orthonormality relations of each species' orbitals, we solve for 
the Lagrange multipliers, $k, j=1, \ldots, M_{A}, k^{\prime}, j^{\prime}=1, \ldots, M_{B}$ and $k^{\prime \prime}, j^{\prime \prime}=1, \ldots, M_{C}$ :

$$
\begin{aligned}
& \mu_{k j}^{(A)}(t)=\left\langle\phi_{j}\left|\sum_{q=1}^{M_{A}}\left(\rho_{k q}^{(A)}\left[\hat{h}^{(A)}-i \frac{\partial}{\partial t}^{(A)}\right]+\left\{\rho_{2} \hat{W}\right\}_{k q}^{(A)}+\left\{\rho_{3} \hat{U}\right\}_{k q}^{(A)}\right)\right| \phi_{q}\right\rangle, \\
& \mu_{k^{\prime} j^{\prime}}^{(B)}(t)=\left\langle\psi_{j^{\prime}}\left|\sum_{q^{\prime}=1}^{M_{B}}\left(\rho_{k^{\prime} q^{\prime}}^{(B)}\left[\hat{h}^{(B)}-i \frac{\partial}{\partial t}^{(B)}\right]+\left\{\rho_{2} \hat{W}\right\}_{k^{\prime} q^{\prime}}^{(B)}+\left\{\rho_{3} \hat{U}\right\}_{k^{\prime} q^{\prime}}^{(B)}\right)\right| \psi_{q^{\prime}}\right\rangle, \\
& \mu_{k^{\prime \prime} j^{\prime \prime}}^{(C)}(t)=\left\langle\chi_{j^{\prime \prime}}\left|\sum_{q^{\prime \prime}=1}^{M_{C}}\left(\rho_{k^{\prime \prime} q^{\prime \prime}}^{(C)}\left[\hat{h}^{(C)}-i \frac{\partial}{\partial t}\right]+\left\{\rho_{2} \hat{W}\right\}_{k^{\prime \prime} q^{\prime \prime}}^{(C)}+\left\{\rho_{3} \hat{U}\right\}_{k^{\prime \prime} q^{\prime \prime}}^{(C)}\right)\right| \chi_{q^{\prime \prime}}\right\rangle .
\end{aligned}
$$

The terms appearing in the Lagrange multipliers are all defined in Appendix C. We discuss them below, after we arrive at the final form of the equations-of-motion for the orbitals.

To proceed we introduce the projection operators for the mixture:

$$
\hat{\mathbf{P}}^{(B)}=1-\sum_{u^{\prime}=1}^{M_{B}}\left|\psi_{u^{\prime}}\right\rangle\left\langle\psi_{u^{\prime}}\left|, \quad \hat{\mathbf{P}}^{(C)}=1-\sum_{u^{\prime \prime}=1}^{M_{C}}\right| \chi_{u^{\prime \prime}}\right\rangle\left\langle\chi_{u^{\prime \prime}}\right|,
$$

where the projection operator for the $A$-species orbitals $\hat{\mathbf{P}}^{(A)}$ was defined in (30). Now, eliminating the Lagrange multipliers (49) and making use of the differential conditions for each species $(25,47)$, we obtain the final form of the equations-of-motion of the orbitals of the mixture, $j=1, \ldots, M_{A}, j^{\prime}=1, \ldots, M_{B}$ and $j^{\prime \prime}=1, \ldots, M_{C}$ :

$$
\begin{aligned}
& i\left|\dot{\phi}_{j}\right\rangle=\hat{\mathbf{P}}^{(A)}\left[\hat{h}^{(A)}\left|\phi_{j}\right\rangle+\sum_{k, q=1}^{M_{A}}\left\{\boldsymbol{\rho}^{(A)}(t)\right\}_{j k}^{-1}\left(\left\{\rho_{2} \hat{W}\right\}_{k q}^{(A)}+\left\{\rho_{3} \hat{U}\right\}_{k q}^{(A)}\right)\left|\phi_{q}\right\rangle\right], \\
& i\left|\dot{\psi}_{j^{\prime}}\right\rangle=\hat{\mathbf{P}}^{(B)}\left[\hat{h}^{(B)}\left|\psi_{j^{\prime}}\right\rangle+\sum_{k^{\prime}, q^{\prime}=1}^{M_{B}}\left\{\boldsymbol{\rho}^{(B)}(t)\right\}_{j^{\prime} k^{\prime}}^{-1}\left(\left\{\rho_{2} \hat{W}\right\}_{k^{\prime} q^{\prime}}^{(B)}+\left\{\rho_{3} \hat{U}\right\}_{k^{\prime} q^{\prime}}^{(B)}\right)\left|\psi_{q^{\prime}}\right\rangle\right], \\
& i\left|\dot{\chi}_{j^{\prime \prime}}\right\rangle=\hat{\mathbf{P}}^{(C)}\left[\hat{h}^{(C)}\left|\chi_{j^{\prime \prime}}\right\rangle+\sum_{k^{\prime \prime}, q^{\prime \prime}=1}^{M_{C}}\left\{\boldsymbol{\rho}^{(C)}(t)\right\}_{j^{\prime \prime} k^{\prime \prime}}^{-1}\left(\left\{\rho_{2} \hat{W}\right\}_{k^{\prime \prime} q^{\prime \prime}}^{(C)}+\left\{\rho_{3} \hat{U}\right\}_{k^{\prime \prime} q^{\prime \prime}}^{(C)}\right)\left|\chi_{q^{\prime \prime}}\right\rangle\right] .
\end{aligned}
$$

We see the appealing structure of the equations-of-motion for the orbitals. The various onebody operators which assemble the contributions from different orders of the interactions, corresponding to the one-, two- and three-body parts of the many-particle Hamiltonian $\hat{H}^{(A B C)}(36)$, are separated. Moreover, it is seen that each one-body operator is comprised of products of reduced density matrices of increasing order times one-body potentials resulting from interactions of the same order (see Appendix C for the explicit terms). This separation, originally put forward for the first time in this context for the single-species static theory for bosons MCHB [56], is not only theoretically appealing, but is expected to make the implementation of the theory in case of higher-body forces further efficient. 
Equations-of-motion (51) for the orbitals together with (48) for the expansion coefficients constitute the propagation theory for mixtures of three kinds of identical particles, interacting with all possible interactions up to three-body forces. All four possible mixtures (FermiFermi-Fermi, Bose-Fermi-Fermi, Bose-Bose-Fermi and Bose-Bose-Bose) are presented in a unified manner, the respective acronyms are denoted as MCTDH-FFF, MCTDH-BFF, MCTDH-BBF and MCTDH-BBB.

To conclude our work, we note that one can compute with imaginary time propagation for time-independent Hamiltonians self-consistent ground and excited states for 3-species mixtures. Substituting $t \rightarrow-i t$ into the equations-of-motion for the coefficients and orbitals, Eqs. $(48,51)$, the final time-independent (static) theory reads, $k=1, \ldots, M_{A}, k^{\prime}=1, \ldots, M_{B}$ and $k^{\prime \prime}=1, \ldots, M_{C}$ :

$$
\begin{gathered}
\sum_{q=1}^{M_{A}}\left[\rho_{k q}^{(A)} \hat{h}^{(A)}+\left\{\rho_{2} \hat{W}\right\}_{k q}^{(A)}+\left\{\rho_{3} \hat{U}\right\}_{k q}^{(A)}\right]\left|\phi_{q}\right\rangle=\sum_{j=1}^{M_{A}} \mu_{k j}^{(A)}\left|\phi_{j}\right\rangle, \\
\sum_{q^{\prime}=1}^{M_{B}}\left[\rho_{k^{\prime} q^{\prime}}^{(B)} \hat{h}^{(B)}+\left\{\rho_{2} \hat{W}\right\}_{k^{\prime} q^{\prime}}^{(B)}+\left\{\rho_{3} \hat{U}\right\}_{k^{\prime} q^{\prime}}^{(B)}\right]\left|\psi_{q^{\prime}}\right\rangle=\sum_{j^{\prime}=1}^{M_{B}} \mu_{k^{\prime} j^{\prime}}^{(B)}\left|\psi_{j^{\prime}}\right\rangle, \\
\sum_{q^{\prime \prime}=1}^{M_{C}}\left[\rho_{k^{\prime \prime} q^{\prime \prime}}^{(C)} \hat{h}^{(C)}+\left\{\rho_{2} \hat{W}\right\}_{k^{\prime \prime} q^{\prime \prime}}^{(C)}+\left\{\rho_{3} \hat{U}\right\}_{k^{\prime \prime} q^{\prime \prime}}^{(C)}\right]\left|\chi_{q^{\prime \prime}}\right\rangle=\sum_{j^{\prime \prime}=1}^{M_{C}} \mu_{k^{\prime \prime} j^{\prime \prime}}^{(C)}\left|\chi_{j^{\prime \prime}}\right\rangle, \\
C_{\vec{J}}^{\hat{H}^{(A B C)}}=\varepsilon^{(A B C)} C_{\vec{J}}, \quad \forall \vec{J},
\end{gathered}
$$

where, making use of the normalization of the static many-particle wave-function $\left|\Psi^{(A B C)}\right\rangle$, $\varepsilon^{(A B C)}=\sum_{\vec{J}} C_{\vec{J}}^{*} C_{\vec{J}}^{\hat{H}^{(A B C)}}$ is the eigen-energy of the system. Finally, utilizing the fact that the matrices of Lagrange multipliers $\left\{\mu_{k j}^{(A)}\right\},\left\{\mu_{k^{\prime} j^{\prime}}^{(B)}\right\}$ and $\left\{\mu_{k^{\prime \prime} j^{\prime \prime}}^{(C)}\right\}$ are Hermitian (for stationary states) and of the invariance property of the multiconfigurational wave-function (to unitary transformations of each species' orbitals compensated by the 'reverse' transformations of the coefficients), one can transform Eq. (52) to a representation where $\left\{\mu_{k j}^{(A)}\right\},\left\{\mu_{k^{\prime} j^{\prime}}^{(B)}\right\}$ and $\left\{\mu_{k^{\prime \prime} j^{\prime \prime}}^{(C)}\right\}$ are diagonal matrices. This concludes our derivations.

\section{BRIEF SUMMARY AND OUTLOOK}

In the present work we have specified the MCTDH method for a new complicated system of relevance. We have considered mixtures of three kinds of identical particles interacting via all combinations of two- and three-body forces. We have derived the equations-of-motion for 
the expansion coefficients, $\left\{C_{\vec{J}}(t)\right\}$, and the orbitals, $\left\{\phi_{k}(\mathbf{x}, t)\right\},\left\{\psi_{k^{\prime}}(\mathbf{y}, t)\right\}$ and $\left\{\chi_{k^{\prime \prime}}(\mathbf{z}, t)\right\}$, see Eqs. (48,51). The self-consistent static theory has been derived as well, see Eq. (52).

All quantities needed for the implementation of the theory have been prescribed in details. On the methodological level, we have represented the coefficients' part of the equations-ofmotion in a compact recursive form in terms of one-body density operators only, $\left\{\hat{\rho}_{k q}^{(A)}\right\}$, $\left\{\hat{\rho}_{k^{\prime} q^{\prime}}^{(B)}\right\}$ and $\left\{\hat{\rho}_{k^{\prime \prime} q^{\prime \prime}}^{(C)}\right\}$. The recursion utilizes the recently proposed Combinadic-based mapping for fermionic and bosonic operators in Fock space [60] that has been successfully applied and implemented within the MCTDHB package [62]. Our derivation sheds new light on the representation of the coefficients' part in MCTDHF and MCTDHB without resorting to the matrix elements of the many-body Hamiltonian with respect to the time-dependent configurations, and suggests a recipe for efficient implementation of MCTDH-FFF, MCTDH-BFF, MCTDH-BBF and MCTDH-BBB which is well-suitable for parallel implementation.

As an outlook of the present theory, let us imagine the possibility of conversion between the distinct particles, say the conversion of the $A$ and $B$ species to the $C$ species, which can be written symbolically as the following "reaction":

$$
A+B \leftrightharpoons C
$$

Such a process would be a model, e.g., for the resonant association of hetero-nuclear ultracold molecules. The derivation of an efficient MCTDH-conversion theory in this case would require the extension of the Combinadic-based mapping [60] to systems with particle conversion, and the assembly of more building bricks than just the one-body density operators used in the present theory, $\left\{\hat{\rho}_{k q}^{(A)}\right\},\left\{\hat{\rho}_{k^{\prime} q^{\prime}}^{(B)}\right\}$ and $\left\{\hat{\rho}_{k^{\prime \prime} q^{\prime \prime}}^{(C)}\right\}$.

\section{Acknowledgments}

The paper is dedicated to Professor Debashis Mukherjee, a dear colleague and friend, on the occasion of his 65th birthday. We are grateful to Hans-Dieter Meyer for multiple and continuous discussions on MCTDH, and acknowledge financial support by the DFG. 


\section{Appendix A: Calculating expectation values of operators in mixtures of three kinds} of identical particles

Following [60], we write the general expectation value of an operator $\hat{O}^{(3 m i x)}$ in a 3 -species mixture as follows:

$$
\begin{aligned}
& \left\langle\Psi^{(A B C)}(t)\left|\hat{O}^{(3 m i x)}\right| \Psi^{(A B C)}(t)\right\rangle=\left\langle\Psi^{(A B C)}(t)\right|\left\{\hat{O}^{(3 m i x)}\left|\Psi^{(A B C)}(t)\right\rangle\right\}= \\
& =\sum_{\vec{J}} C_{\vec{J}}^{*}(t) C_{\vec{J}}^{\hat{O}^{(3 m i x)}}(t),
\end{aligned}
$$

where

$$
\hat{O}^{(3 m i x)}\left|\Psi^{(A B C)}(t)\right\rangle=\hat{O}^{(3 m i x)} \sum_{\vec{J}} C_{\vec{J}}(t)|\vec{J} ; t\rangle \equiv \sum_{\vec{J}} C_{\vec{J}}^{\hat{O}^{(3 m i x)}}(t)|\vec{J} ; t\rangle .
$$

$\hat{O}^{(3 m i x)}$ can be a one-, two- or three-body operator or any combination thereof.

The operation of single-species operators, whether $\hat{O}^{(A)}, \hat{O}^{(B)}$ or $\hat{O}^{(C)}$ can be read of directly from Eqs. (11-19) and we will not repeat them here (one needs just to replace therein $J_{A}$ by $\vec{J}$ in the overall notation, and $M_{A}$ by $M_{B}$ or $M_{C}$, when appropriate; also see $[60])$.

For the inter-species two-body operators we prescribe the compact result for completeness. For the two-body operators $\hat{O}^{(A B)}=\sum_{k, k^{\prime}, q, q^{\prime}} O_{k k^{\prime} q q^{\prime}}^{(A B)} \hat{\rho}_{k q}^{(A)} \hat{\rho}_{k^{\prime} q^{\prime}}^{(B)}$, $\hat{O}^{(A C)}=\sum_{k, k^{\prime \prime}, q, q^{\prime \prime}} O_{k k^{\prime \prime} q q^{\prime \prime}}^{(A C)} \hat{\rho}_{k q}^{(A)} \hat{\rho}_{k^{\prime \prime} q^{\prime \prime}}^{(C)}$ and $\hat{O}^{(B C)}=\sum_{k^{\prime}, k^{\prime \prime}, q^{\prime}, q^{\prime \prime}} O_{k^{\prime} k^{\prime \prime} q^{\prime} q^{\prime \prime}}^{(B C)} \hat{\rho}_{k^{\prime} q^{\prime}}^{(B)} \hat{\rho}_{k^{\prime \prime} q^{\prime \prime}}^{(C)}$ we find:

$$
\begin{aligned}
& C_{\vec{J}}^{\hat{O}^{(A B)}}(t)=\sum_{k, k^{\prime}, q, q^{\prime}=1}^{M_{A}, M_{B}} O_{k k^{\prime} q q^{\prime}}^{(A B)} C_{\vec{J}}^{\hat{\rho}_{k q}^{(A)} \hat{\rho}_{k^{\prime} q^{\prime}}^{(B)}(t),} \\
& C_{\vec{J}}^{\hat{O}^{(A C)}}(t)=\sum_{k, k^{\prime \prime}, q, q^{\prime \prime}=1}^{M_{A}, M_{C}} O_{k k^{\prime \prime} q q^{\prime \prime}}^{(A C)} C_{\vec{J}}^{\hat{\rho}_{k q}^{(A)} \hat{\rho}_{k^{\prime \prime} q^{\prime \prime}}^{(C)}(t),} \\
& C_{\vec{J}}^{\hat{O}^{(B C)}}(t)=\sum_{k^{\prime}, k^{\prime \prime}, q^{\prime}, q^{\prime \prime}=1}^{M_{B}, M_{C}} O_{k^{\prime} k^{\prime \prime} q^{\prime} q^{\prime \prime}}^{(B C)} C_{\vec{J}}^{\hat{\rho}_{k^{\prime} q^{\prime}}^{(B)} \hat{\rho}_{k^{\prime \prime} q^{\prime \prime}}^{(C)}(t) .}
\end{aligned}
$$

Note the factorization of the one-body (basic) density operators for the inter-species operators, which simplify the way how the coefficients' vector is evaluated.

For the inter-species three-body operators resulting from the force between two identical particles and a third distinct one we list the final result for completeness. For the three-body 
operators

$$
\begin{aligned}
\hat{O}^{(A A B)} & =\frac{1}{2} \sum_{k, k^{\prime}, s, q, q^{\prime} l} O_{k k^{\prime} s q q^{\prime} l}^{(A A B)} \hat{\rho}_{k s l q}^{(A)} \hat{\rho}_{k^{\prime} q^{\prime}}^{(B)}, \\
\hat{O}^{(A B B)} & =\frac{1}{2} \sum_{k, k^{\prime}, s^{\prime}, q, q^{\prime}, l^{\prime}} O_{k k^{\prime} s^{\prime} q q^{\prime} l^{\prime}}^{(A B B)} \hat{\rho}_{k q}^{(A)} \hat{\rho}_{k^{\prime} s^{\prime} l^{\prime} q^{\prime}}^{(B)}, \\
\hat{O}^{(A A C)} & =\frac{1}{2} \sum_{k, k^{\prime \prime}, s, q, q^{\prime \prime} l} O_{k k^{\prime \prime} s q q^{\prime \prime} l}^{(A A C)} \hat{\rho}_{k s l q}^{(A)} \hat{\rho}_{k^{\prime \prime} q^{\prime \prime}}^{(C)}, \\
\hat{O}^{(A C C)} & =\frac{1}{2} \sum_{k, k^{\prime \prime}, s^{\prime \prime}, q, q^{\prime \prime}, l^{\prime \prime}} O_{k k^{\prime \prime} s^{\prime \prime} q q^{\prime \prime} l^{\prime \prime}}^{(A C C)} \hat{\rho}_{k q}^{(A)} \hat{\rho}_{k^{\prime \prime} s^{\prime \prime} l^{\prime \prime} q^{\prime \prime}}^{(C)}, \\
\hat{O}^{(B B C)} & =\frac{1}{2} \sum_{k^{\prime}, k^{\prime \prime}, s^{\prime}, q^{\prime}, q^{\prime \prime} l^{\prime}} O_{k^{\prime} k^{\prime \prime} s^{\prime} q^{\prime} q^{\prime \prime} l^{\prime}}^{(B B C)} \hat{\rho}_{k^{\prime} s^{\prime} l^{\prime} q^{\prime}}^{(B)} \hat{\rho}_{k^{\prime \prime} q^{\prime \prime}}^{(C)}, \\
\hat{O}^{(B C C)} & =\frac{1}{2} \sum_{k^{\prime}, k^{\prime \prime}, s^{\prime \prime}, q^{\prime}, q^{\prime \prime}, l^{\prime \prime}} O_{k^{\prime} k^{\prime \prime} s^{\prime \prime} q^{\prime} q^{\prime \prime} l^{\prime \prime}}^{\left(B C \hat{\rho}_{k^{\prime} q^{\prime}}^{(B)} \hat{\rho}_{k^{\prime \prime} s^{\prime \prime} l^{\prime \prime} q^{\prime \prime}}^{(C)},\right.}
\end{aligned}
$$


we find:

$$
\begin{aligned}
& C_{\vec{J}}^{\hat{O}^{(A A B)}}(t)=
\end{aligned}
$$

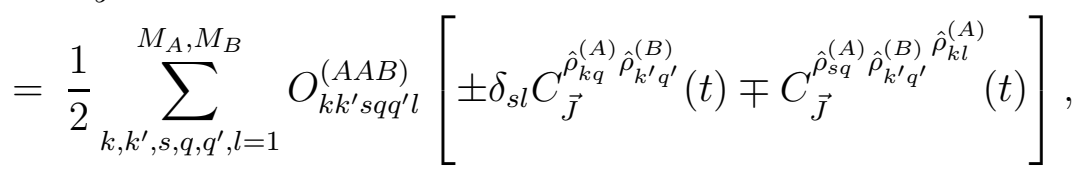

$$
\begin{aligned}
& C_{\vec{J}}^{\hat{O}^{(A B B)}}(t)=
\end{aligned}
$$

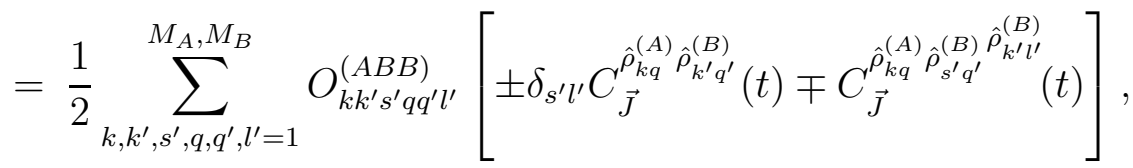

$$
\begin{aligned}
& C_{\vec{J}}^{\hat{O}^{(A A C)}}(t)=
\end{aligned}
$$

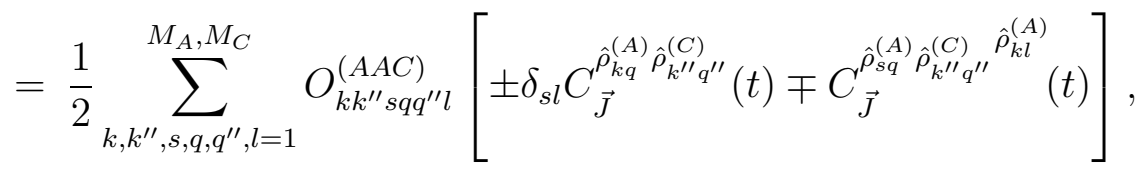

$$
\begin{aligned}
& C_{\vec{J}}^{\hat{O}^{(A C C)}}(t)=
\end{aligned}
$$

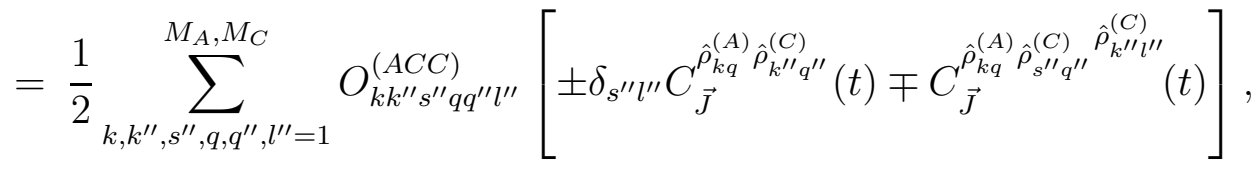

$$
\begin{aligned}
& C_{\vec{J}}^{\hat{O}^{(B B C)}}(t)=
\end{aligned}
$$

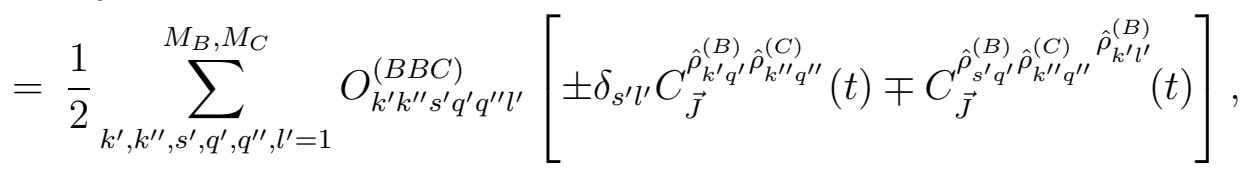

$$
\begin{aligned}
& C_{\vec{J}}^{\hat{O}^{(B C C)}}(t)=
\end{aligned}
$$

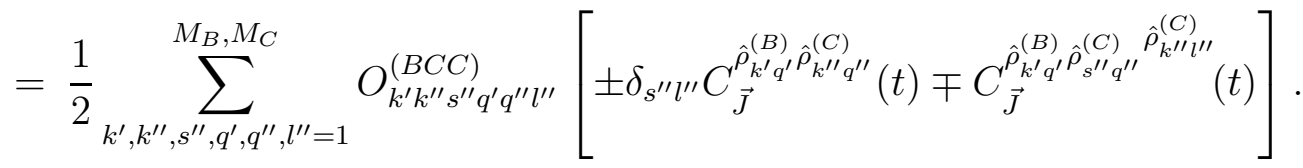

We remind that the appearance of the one-body (basic) density operators on two levels means that the lower-level multiplication has to be performed first, and the upper-level second.

Finally, for the inter-species three-body operator we give the closed-form result for completeness. For the three-body operator $\hat{O}^{(A B C)}=\sum_{k, k^{\prime}, k^{\prime \prime}, q, q^{\prime}, q^{\prime \prime}} O_{k k^{\prime} k^{\prime \prime} q q^{\prime} q^{\prime \prime}}^{(A B C)} \hat{\rho}_{k q}^{(A)} \hat{\rho}_{k^{\prime} q^{\prime}}^{(B)} \hat{\rho}_{k^{\prime \prime} q^{\prime \prime}}^{(C)}$ we find:

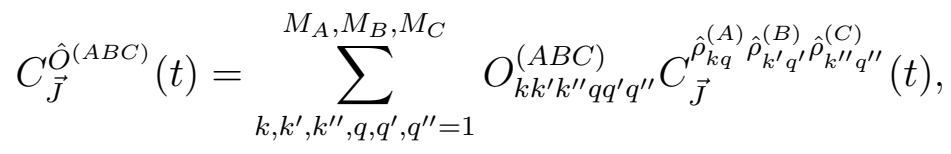

which concludes our Combinadic-based [60] representation of the equations-of-motion for the coefficients in MCTDH for mixtures of 3 kinds of identical particles interacting with 
up to 3-body forces, and the calculations of all relevant matrix elements with respect to $\left|\Psi^{(A B C)}(t)\right\rangle$.

Appendix B: Reduced density matrices for mixtures of three kinds of identical particles interacting with up to three-body forces

\section{Intra-species reduced density matrices}

The reduced one-body density matrix of the single-species multiconfigurational wavefunction $\left|\Psi^{(A)}(t)\right\rangle$ is given by:

$$
\begin{aligned}
& \rho^{(A)}\left(\mathbf{x}_{1} \mid \mathbf{x}_{1}^{\prime} ; t\right)=N_{A} \int d \mathbf{x}_{2} d \mathbf{x}_{3} \cdots d \mathbf{x}_{N_{A}} \\
& =\Psi^{(A)^{*}}\left(\mathbf{x}_{1}^{\prime}, \mathbf{x}_{2}, \ldots, \mathbf{x}_{N_{A}} ; t\right) \Psi^{(A)}\left(\mathbf{x}_{1}, \mathbf{x}_{2}, \ldots, \mathbf{x}_{N_{A}} ; t\right)= \\
& =\left\langle\Psi^{(A)}(t)\right|\left\{\hat{\Psi}_{A}^{\dagger}\left(\mathbf{x}_{1}^{\prime}\right) \hat{\Psi}_{A}\left(\mathbf{x}_{1}\right)\left|\Psi^{(A)}(t)\right\rangle\right\}=\sum_{k, q=1}^{M} \rho_{k q}^{(A)}(t) \phi_{k}^{*}\left(\mathbf{x}_{1}^{\prime}, t\right) \phi_{q}\left(\mathbf{x}_{1}, t\right),
\end{aligned}
$$

where its matrix elements in the orbital basis $\rho_{k q}^{(A)}(t)$ are given in Eq. (28) of the main text.

Then, the reduced two-body density matrix of the single-species multiconfigurational wave-function $\left|\Psi^{(A)}(t)\right\rangle$ is given by:

$$
\begin{aligned}
& \rho^{(A)}\left(\mathbf{x}_{1}, \mathbf{x}_{2} \mid \mathbf{x}_{1}^{\prime}, \mathbf{x}_{2}^{\prime} ; t\right)=N_{A}\left(N_{A}-1\right) \int d \mathbf{x}_{3} \cdots d \mathbf{x}_{N_{A}} \times \\
& \times \Psi^{(A)^{*}}\left(\mathbf{x}_{1}^{\prime}, \mathbf{x}_{2}^{\prime}, \mathbf{x}_{3}, \ldots, \mathbf{x}_{N_{A}} ; t\right) \Psi^{(A)}\left(\mathbf{x}_{1}, \mathbf{x}_{2}, \mathbf{x}_{3}, \ldots, \mathbf{x}_{N_{A}} ; t\right)= \\
& =\left\langle\Psi^{(A)}(t)\right|\left\{\hat{\Psi}_{A}^{\dagger}\left(\mathbf{x}_{1}^{\prime}\right) \hat{\Psi}_{A}^{\dagger}\left(\mathbf{x}_{2}^{\prime}\right) \hat{\Psi}_{A}\left(\mathbf{x}_{2}\right) \hat{\Psi}_{A}\left(\mathbf{x}_{1}\right)\left|\Psi^{(A)}(t)\right\rangle\right\}= \\
& =\sum_{k, s, l, q=1}^{M} \rho_{k s l q}^{(A)}(t) \phi_{k}^{*}\left(\mathbf{x}_{1}^{\prime}, t\right) \phi_{s}^{*}\left(\mathbf{x}_{2}^{\prime}, t\right) \phi_{l}\left(\mathbf{x}_{2}, t\right) \phi_{q}\left(\mathbf{x}_{1}, t\right),
\end{aligned}
$$

where its matrix elements in the orbital basis $\rho_{k s l q}^{(A)}(t)$ are given in Eq. (28).

Finally in the single-species case, the reduced three-body density matrix of $\left|\Psi^{(A)}(t)\right\rangle$ is given by:

$$
\begin{aligned}
& \rho^{(A)}\left(\mathbf{x}_{1}, \mathbf{x}_{2}, \mathbf{x}_{3} \mid \mathbf{x}_{1}^{\prime}, \mathbf{x}_{2}^{\prime}, \mathbf{x}_{3}^{\prime} ; t\right)=N_{A}\left(N_{A}-1\right)\left(N_{A}-2\right) \int d \mathbf{x}_{4} \cdots d \mathbf{x}_{N_{A}} \times \\
& \times \Psi^{(A)^{*}}\left(\mathbf{x}_{1}^{\prime}, \mathbf{x}_{2}^{\prime}, \mathbf{x}_{3}^{\prime}, \mathbf{x}_{4}, \ldots, \mathbf{x}_{N_{A}} ; t\right) \Psi^{(A)}\left(\mathbf{x}_{1}, \mathbf{x}_{2}, \mathbf{x}_{3}, \mathbf{x}_{4}, \ldots, \mathbf{x}_{N_{A}} ; t\right)= \\
& =\left\langle\Psi^{(A)}(t)\right|\left\{\hat{\Psi}_{A}^{\dagger}\left(\mathbf{x}_{1}^{\prime}\right) \hat{\mathbf{\Psi}}_{A}^{\dagger}\left(\mathbf{x}_{2}^{\prime}\right) \hat{\Psi}_{A}^{\dagger}\left(\mathbf{x}_{3}^{\prime}\right) \hat{\mathbf{\Psi}}_{A}\left(\mathbf{x}_{3}\right) \hat{\mathbf{\Psi}}_{A}\left(\mathbf{x}_{2}\right) \hat{\mathbf{\Psi}}_{A}\left(\mathbf{x}_{1}\right)\left|\Psi^{(A)}(t)\right\rangle\right\}= \\
& =\sum_{k, s, p, r, l, q=1}^{M} \rho_{k s p r l q}^{(A)}(t) \phi_{k}^{*}\left(\mathbf{x}_{1}^{\prime}, t\right) \phi_{s}^{*}\left(\mathbf{x}_{2}^{\prime}, t\right) \phi_{p}^{*}\left(\mathbf{x}_{3}^{\prime}, t\right) \phi_{r}\left(\mathbf{x}_{3}, t\right) \phi_{l}\left(\mathbf{x}_{2}, t\right) \phi_{q}\left(\mathbf{x}_{1}, t\right),
\end{aligned}
$$


where its matrix elements in the orbital basis $\rho_{k s p r l q}^{(A)}(t)$ are given in Eq. (28).

The reduced density matrices of the $B$ and $C$ species are defined in an analogous manner, where $B$ and $C$ quantities are to replace the $A$ quantities in Eqs. (B1-B3).

\section{Inter-species reduced two-body density matrices}

For completeness, we give all inter-species reduced density matrices that occur in a mixture of three kinds of identical particles interacting with up to three-body forces, where each species may have a different spin. There are three such reduced density matrices which are associated with the two-body interactions of two distinct particles.

$$
\begin{aligned}
& \rho^{(A B)}\left(\mathbf{x}_{1}, \mathbf{y}_{1} \mid \mathbf{x}_{1}^{\prime}, \mathbf{y}_{1}^{\prime} ; t\right)=N_{A} N_{B} \int d \mathbf{x}_{2} \cdots d \mathbf{x}_{N_{A}} d \mathbf{y}_{2} \cdots d \mathbf{y}_{N_{B}} d \mathbf{z}_{1} \cdots d \mathbf{z}_{N_{C}} \times \\
& \times \Psi^{(A B C)^{*}}\left(\mathbf{x}_{1}^{\prime}, \ldots, \mathbf{x}_{N_{A}}, \mathbf{y}_{1}^{\prime}, \ldots, \mathbf{y}_{N_{B}}, \mathbf{z}_{1}, \ldots, \mathbf{z}_{N_{C}} ; t\right) \times \\
& \times \Psi^{(A B C)}\left(\mathbf{x}_{1}, \ldots, \mathbf{x}_{N_{A}}, \mathbf{y}_{1}, \ldots, \mathbf{y}_{N_{B}}, \mathbf{z}_{1}, \ldots, \mathbf{z}_{N_{C}} ; t\right)= \\
& =\left\langle\Psi^{(A B C)}(t)\right|\left\{\hat{\mathbf{\Psi}}_{A}^{\dagger}\left(\mathbf{x}_{1}^{\prime}\right) \hat{\mathbf{\Psi}}_{A}\left(\mathbf{x}_{1}\right) \hat{\mathbf{\Psi}}_{B}^{\dagger}\left(\mathbf{y}_{1}^{\prime}\right) \hat{\mathbf{\Psi}}_{B}\left(\mathbf{y}_{1}\right)\left|\Psi^{(A B C)}(t)\right\rangle\right\}= \\
& =\sum_{k, k^{\prime}, q, q^{\prime}=1}^{M_{A}, M_{B}} \rho_{k k^{\prime} q q^{\prime}}^{(A B)}(t) \phi_{k}^{*}\left(\mathbf{x}_{1}^{\prime}, t\right) \phi_{q}\left(\mathbf{x}_{1}, t\right) \psi_{k^{\prime}}^{*}\left(\mathbf{y}_{1}^{\prime}, t\right) \psi_{q^{\prime}}\left(\mathbf{y}_{1}, t\right),
\end{aligned}
$$

where its matrix elements in the orbital basis are give by:

$$
\begin{gathered}
\rho_{k k^{\prime} q q^{\prime}}^{(A B)}(t)=\sum_{\vec{J}} C_{\vec{J}}^{*}(t) C_{\vec{J}}^{\hat{\rho}_{k q}^{(A)} \hat{\rho}_{k^{\prime} q^{\prime}}^{(B)}(t) .} \\
\rho^{(A C)}\left(\mathbf{x}_{1}, \mathbf{z}_{1} \mid \mathbf{x}_{1}^{\prime}, \mathbf{z}_{1}^{\prime} ; t\right)=N_{A} N_{C} \int d \mathbf{x}_{2} \cdots d \mathbf{x}_{N_{A}} d \mathbf{y}_{1} \cdots d \mathbf{y}_{N_{B}} d \mathbf{z}_{2} \cdots d \mathbf{z}_{N_{C}} \times \\
\times \Psi^{(A B C)^{*}}\left(\mathbf{x}_{1}^{\prime}, \ldots, \mathbf{x}_{N_{A}}, \mathbf{y}_{1}, \ldots, \mathbf{y}_{N_{B}}, \mathbf{z}_{1}^{\prime}, \ldots, \mathbf{z}_{N_{C}} ; t\right) \times \\
\times \Psi^{(A B C)}\left(\mathbf{x}_{1}, \ldots, \mathbf{x}_{N_{A}}, \mathbf{y}_{1}, \ldots, \mathbf{y}_{N_{B}}, \mathbf{z}_{1}, \ldots, \mathbf{z}_{N_{C}} ; t\right)= \\
=\left\langle\Psi^{(A B C)}(t)\right|\left\{\hat{\Psi}_{A}^{\dagger}\left(\mathbf{x}_{1}^{\prime}\right) \hat{\mathbf{\Psi}}_{A}\left(\mathbf{x}_{1}\right) \hat{\Psi}_{C}^{\dagger}\left(\mathbf{z}_{1}^{\prime}\right) \hat{\Psi}_{C}\left(\mathbf{z}_{1}\right)\left|\Psi^{(A B C)}(t)\right\rangle\right\}= \\
=\sum_{k, k^{\prime \prime}, q, q^{\prime \prime}=1}^{M_{A}, M_{B}} \rho_{k k^{\prime \prime} q q^{\prime \prime}}^{(A C)}(t) \phi_{k}^{*}\left(\mathbf{x}_{1}^{\prime}, t\right) \phi_{q}\left(\mathbf{x}_{1}, t\right) \chi_{k^{\prime \prime}}^{*}\left(\mathbf{z}_{1}^{\prime}, t\right) \chi_{q^{\prime \prime}}\left(\mathbf{z}_{1}, t\right),
\end{gathered}
$$

where its matrix elements in the orbital basis are given by:

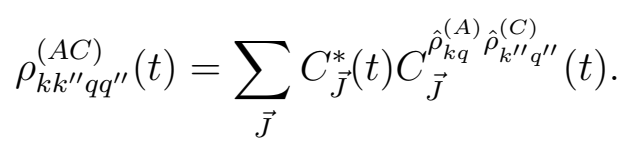




$$
\begin{aligned}
& \rho^{(B C)}\left(\mathbf{y}_{1}, \mathbf{z}_{1} \mid \mathbf{y}_{1}^{\prime}, \mathbf{z}_{1}^{\prime} ; t\right)=N_{B} N_{C} \int d \mathbf{x}_{1} \cdots d \mathbf{x}_{N_{A}} d \mathbf{y}_{2} \cdots d \mathbf{y}_{N_{B}} d \mathbf{z}_{2} \cdots d \mathbf{z}_{N_{C}} \times \\
& \times \Psi^{(A B C)^{*}}\left(\mathbf{x}_{1}, \ldots, \mathbf{x}_{N_{A}}, \mathbf{y}_{1}^{\prime}, \ldots, \mathbf{y}_{N_{B}}, \mathbf{z}_{1}^{\prime}, \ldots, \mathbf{z}_{N_{C}} ; t\right) \times \\
& \times \Psi^{(A B C)}\left(\mathbf{x}_{1}, \ldots, \mathbf{x}_{N_{A}}, \mathbf{y}_{1}, \ldots, \mathbf{y}_{N_{B}}, \mathbf{z}_{1}, \ldots, \mathbf{z}_{N_{C}} ; t\right)= \\
& =\left\langle\Psi^{(A B C)}(t)\right|\left\{\hat{\mathbf{\Psi}}_{B}^{\dagger}\left(\mathbf{y}_{1}^{\prime}\right) \hat{\mathbf{\Psi}}_{B}\left(\mathbf{y}_{1}\right) \hat{\mathbf{\Psi}}_{C}^{\dagger}\left(\mathbf{z}_{1}^{\prime}\right) \hat{\mathbf{\Psi}}_{C}\left(\mathbf{z}_{1}\right)\left|\Psi^{(A B C)}(t)\right\rangle\right\}= \\
& =\sum_{k^{\prime}, k^{\prime \prime}, q^{\prime}, q^{\prime \prime}=1}^{M_{B}, M_{B}} \rho_{k^{\prime} k^{\prime \prime} q^{\prime} q^{\prime \prime}}^{(B C)}(t) \psi_{k^{\prime}}^{*}\left(\mathbf{y}_{1}^{\prime}, t\right) \psi_{q^{\prime}}\left(\mathbf{y}_{1}, t\right) \chi_{k^{\prime \prime}}^{*}\left(\mathbf{z}_{1}^{\prime}, t\right) \chi_{q^{\prime \prime}}\left(\mathbf{z}_{1}, t\right),
\end{aligned}
$$

where its matrix elements in the orbital basis are given by:

$$
\rho_{k^{\prime} k^{\prime \prime} q^{\prime} q^{\prime \prime}}^{(A C)}(t)=\sum_{\vec{J}} C_{\vec{J}}^{*}(t) C_{\vec{J}}^{\hat{\rho}_{k^{\prime} q^{\prime}}^{(B)} \hat{\rho}_{k^{\prime \prime} q^{\prime \prime}}^{(C)}}(t) .
$$

\section{Inter-species reduced three-body density matrices}

There are six reduced three-body density matrices which are associated with the three-

body interactions of two identical particles with a third distinct one.

$$
\begin{aligned}
& \rho^{(A A B)}\left(\mathbf{x}_{1}, \mathbf{x}_{2}, \mathbf{y}_{1} \mid \mathbf{x}_{1}^{\prime}, \mathbf{x}_{2}^{\prime}, \mathbf{y}_{1}^{\prime} ; t\right)= \\
& =N_{A}\left(N_{A}-1\right) N_{B} \int d \mathbf{x}_{3} \cdots d \mathbf{x}_{N_{A}} d \mathbf{y}_{2} \cdots d \mathbf{y}_{N_{B}} d \mathbf{z}_{1} \cdots d \mathbf{z}_{N_{C}} \times \\
& \times \Psi^{(A B C)^{*}}\left(\mathbf{x}_{1}^{\prime}, \mathbf{x}_{2}^{\prime}, \ldots, \mathbf{x}_{N_{A}}, \mathbf{y}_{1}^{\prime}, \mathbf{y}_{2}, \ldots, \mathbf{y}_{N_{B}}, \mathbf{z}_{1}, \mathbf{z}_{2}, \ldots, \mathbf{z}_{N_{C}} ; t\right) \times \\
& \times \Psi^{(A B C)}\left(\mathbf{x}_{1}, \mathbf{x}_{2}, \ldots, \mathbf{x}_{N_{A}}, \mathbf{y}_{1}, \mathbf{y}_{2}, \ldots, \mathbf{y}_{N_{B}}, \mathbf{z}_{1}, \mathbf{z}_{2}, \ldots, \mathbf{z}_{N_{C}} ; t\right)= \\
& =\left\langle\Psi^{(A B C)}(t)\right|\left\{\hat{\mathbf{\Psi}}_{A}^{\dagger}\left(\mathbf{x}_{1}^{\prime}\right) \hat{\mathbf{\Psi}}_{A}^{\dagger}\left(\mathbf{x}_{2}^{\prime}\right) \hat{\mathbf{\Psi}}_{A}\left(\mathbf{x}_{2}\right) \hat{\mathbf{\Psi}}_{A}\left(\mathbf{x}_{1}\right) \hat{\mathbf{\Psi}}_{B}^{\dagger}\left(\mathbf{y}_{1}^{\prime}\right) \hat{\mathbf{\Psi}}_{B}\left(\mathbf{y}_{1}\right)\left|\Psi^{(A B C)}(t)\right\rangle\right\}= \\
& =\sum_{k, k^{\prime}, s, l, q, q^{\prime}=1}^{M_{A}, M_{B}} \rho_{k k^{\prime} s l q q^{\prime}}^{(A A B)}(t) \phi_{k}^{*}\left(\mathbf{x}_{1}^{\prime}, t\right) \phi_{s}^{*}\left(\mathbf{x}_{2}^{\prime}, t\right) \phi_{l}\left(\mathbf{x}_{2}, t\right) \phi_{q}\left(\mathbf{x}_{1}, t\right) \psi_{k^{\prime}}^{*}\left(\mathbf{y}_{1}^{\prime}, t\right) \psi_{q^{\prime}}\left(\mathbf{y}_{1}, t\right),
\end{aligned}
$$

where

$$
\rho_{k k^{\prime} s l q q^{\prime}}^{(A A B)}(t)=\sum_{\vec{J}} C_{\vec{J}}^{*}(t) C_{\vec{J}}^{\hat{\rho}_{k s l q}^{(A)} \hat{\rho}_{k^{\prime} q^{\prime}}^{(B)}}(t)
$$

are its matrix elements in the orbital basis. 


$$
\begin{aligned}
& \rho^{(A B B)}\left(\mathbf{x}_{1}, \mathbf{y}_{1}, \mathbf{y}_{2} \mid \mathbf{x}_{1}^{\prime}, \mathbf{y}_{1}^{\prime}, \mathbf{y}_{2}^{\prime} ; t\right)= \\
= & N_{A} N_{B}\left(N_{B}-1\right) \int d \mathbf{x}_{2} \cdots d \mathbf{x}_{N_{A}} d \mathbf{y}_{3} \cdots d \mathbf{y}_{N_{B}} d \mathbf{z}_{1} \cdots d \mathbf{z}_{N_{C}} \times \\
\times & \Psi^{(A B C)^{*}}\left(\mathbf{x}_{1}^{\prime}, \mathbf{x}_{2}, \ldots, \mathbf{x}_{N_{A}}, \mathbf{y}_{1}^{\prime}, \mathbf{y}_{2}^{\prime}, \ldots, \mathbf{y}_{N_{B}}, \mathbf{z}_{1}, \mathbf{z}_{2}, \ldots, \mathbf{z}_{N_{C}} ; t\right) \times \\
\times & \Psi^{(A B C)}\left(\mathbf{x}_{1}, \mathbf{x}_{2}, \ldots, \mathbf{x}_{N_{A}}, \mathbf{y}_{1}, \mathbf{y}_{2}, \ldots, \mathbf{y}_{N_{B}}, \mathbf{z}_{1}, \mathbf{z}_{2}, \ldots, \mathbf{z}_{N_{C}} ; t\right)= \\
= & \left\langle\Psi^{(A B C)}(t)\right|\left\{\hat{\mathbf{\Psi}}_{A}^{\dagger}\left(\mathbf{x}_{1}^{\prime}\right) \hat{\mathbf{\Psi}}_{A}\left(\mathbf{x}_{1}\right) \hat{\mathbf{\Psi}}_{B}^{\dagger}\left(\mathbf{y}_{1}^{\prime}\right) \hat{\mathbf{\Psi}}_{B}^{\dagger}\left(\mathbf{y}_{2}^{\prime}\right) \hat{\mathbf{\Psi}}_{B}\left(\mathbf{y}_{2}\right) \hat{\mathbf{\Psi}}_{B}\left(\mathbf{y}_{1}\right)\left|\Psi^{(A B C)}(t)\right\rangle\right\}= \\
= & \sum_{k, k^{\prime}, s^{\prime}, l^{\prime}, q, q^{\prime}=1}^{M_{A}, M_{B}} \rho_{k k^{\prime} s^{\prime} l^{\prime} q q^{\prime}}^{(A B B)}(t) \phi_{k}^{*}\left(\mathbf{x}_{1}^{\prime}, t\right) \phi_{q}\left(\mathbf{x}_{1}, t\right) \psi_{k^{\prime}}^{*}\left(\mathbf{y}_{1}^{\prime}, t\right) \psi_{s^{\prime}}^{*}\left(\mathbf{y}_{2}^{\prime}, t\right) \psi_{l^{\prime}}\left(\mathbf{y}_{2}, t\right) \psi_{q^{\prime}}\left(\mathbf{y}_{1}, t\right),
\end{aligned}
$$

where

$$
\rho_{k k^{\prime} s^{\prime} l^{\prime} q q^{\prime}}^{(A B B)}(t)=\sum_{\vec{J}} C_{\vec{J}}^{*}(t) C_{\vec{J}}^{\hat{\rho}_{k q}^{(A)}} \hat{\rho}_{k^{\prime} s^{\prime} l^{\prime} q^{\prime}}^{(B)}(t)
$$

are its matrix elements in the orbital basis.

$$
\begin{aligned}
& \rho^{(A A C)}\left(\mathbf{x}_{1}, \mathbf{x}_{2}, \mathbf{z}_{1} \mid \mathbf{x}_{1}^{\prime}, \mathbf{x}_{2}^{\prime}, \mathbf{z}_{1}^{\prime} ; t\right)= \\
& =N_{A}\left(N_{A}-1\right) N_{C} \int d \mathbf{x}_{3} \cdots d \mathbf{x}_{N_{A}} d \mathbf{y}_{1} \cdots d \mathbf{y}_{N_{B}} d \mathbf{z}_{2} \cdots d \mathbf{z}_{N_{C}} \times \\
& \times \Psi^{(A B C)^{*}}\left(\mathbf{x}_{1}^{\prime}, \mathbf{x}_{2}^{\prime}, \ldots, \mathbf{x}_{N_{A}}, \mathbf{y}_{1}, \mathbf{y}_{2}, \ldots, \mathbf{y}_{N_{B}}, \mathbf{z}_{1}^{\prime}, \mathbf{z}_{2}, \ldots, \mathbf{z}_{N_{C}} ; t\right) \times \\
& \times \Psi^{(A B C)}\left(\mathbf{x}_{1}, \mathbf{x}_{2}, \ldots, \mathbf{x}_{N_{A}}, \mathbf{y}_{1}, \mathbf{y}_{2}, \ldots, \mathbf{y}_{N_{B}}, \mathbf{z}_{1}, \mathbf{z}_{2}, \ldots, \mathbf{z}_{N_{C}} ; t\right)= \\
& =\left\langle\Psi^{(A B C)}(t)\right|\left\{\hat{\Psi}_{A}^{\dagger}\left(\mathbf{x}_{1}^{\prime}\right) \hat{\Psi}_{A}^{\dagger}\left(\mathbf{x}_{2}^{\prime}\right) \hat{\mathbf{\Psi}}_{A}\left(\mathbf{x}_{2}\right) \hat{\mathbf{\Psi}}_{A}\left(\mathbf{x}_{1}\right) \hat{\mathbf{\Psi}}_{C}^{\dagger}\left(\mathbf{z}_{1}^{\prime}\right) \hat{\mathbf{\Psi}}_{C}\left(\mathbf{z}_{1}\right)\left|\Psi^{(A B C)}(t)\right\rangle\right\}= \\
& =\sum_{k, k^{\prime \prime}, s, l, q, q^{\prime \prime}=1}^{M_{A}, M_{C}} \rho_{k k^{\prime \prime} s l q q^{\prime \prime}}^{(A A C)}(t) \phi_{k}^{*}\left(\mathbf{x}_{1}^{\prime}, t\right) \phi_{s}^{*}\left(\mathbf{x}_{2}^{\prime}, t\right) \phi_{l}\left(\mathbf{x}_{2}, t\right) \phi_{q}\left(\mathbf{x}_{1}, t\right) \chi_{k^{\prime \prime}}^{*}\left(\mathbf{z}_{1}^{\prime}, t\right) \chi_{q^{\prime \prime}}\left(\mathbf{z}_{1}, t\right),
\end{aligned}
$$

where

$$
\rho_{k k^{\prime \prime} s l q q^{\prime \prime}}^{(A A C)}(t)=\sum_{\vec{J}} C_{\vec{J}}^{*}(t) C_{\vec{J}}^{\hat{\rho}_{s s l q}^{(A)} \hat{\rho}_{k^{\prime \prime} q^{\prime \prime}}^{(C)}}(t)
$$

are its matrix elements in the orbital basis. 


$$
\begin{aligned}
& \rho^{(A C C)}\left(\mathbf{x}_{1}, \mathbf{z}_{1}, \mathbf{z}_{2} \mid \mathbf{x}_{1}^{\prime}, \mathbf{z}_{1}^{\prime}, \mathbf{z}_{2}^{\prime} ; t\right)= \\
& =N_{A} N_{C}\left(N_{C}-1\right) \int d \mathbf{x}_{2} \cdots d \mathbf{x}_{N_{A}} d \mathbf{y}_{1} \cdots d \mathbf{y}_{N_{B}} d \mathbf{z}_{3} \cdots d \mathbf{z}_{N_{C}} \times \\
& \times \Psi^{(A B C)^{*}}\left(\mathbf{x}_{1}^{\prime}, \mathbf{x}_{2}, \ldots, \mathbf{x}_{N_{A}}, \mathbf{y}_{1}, \mathbf{y}_{2}, \ldots, \mathbf{y}_{N_{B}}, \mathbf{z}_{1}^{\prime}, \mathbf{z}_{2}^{\prime}, \ldots, \mathbf{z}_{N_{C}} ; t\right) \times \\
& \times \Psi^{(A B C)}\left(\mathbf{x}_{1}, \mathbf{x}_{2}, \ldots, \mathbf{x}_{N_{A}}, \mathbf{y}_{1}, \mathbf{y}_{2}, \ldots, \mathbf{y}_{N_{B}}, \mathbf{z}_{1}, \mathbf{z}_{2}, \ldots, \mathbf{z}_{N_{C}} ; t\right)= \\
& =\left\langle\Psi^{(A B C)}(t)\right|\left\{\hat{\Psi}_{A}^{\dagger}\left(\mathbf{x}_{1}^{\prime}\right) \hat{\mathbf{\Psi}}_{A}\left(\mathbf{x}_{1}\right) \hat{\mathbf{\Psi}}_{C}^{\dagger}\left(\mathbf{z}_{1}^{\prime}\right) \hat{\mathbf{\Psi}}_{C}^{\dagger}\left(\mathbf{z}_{2}^{\prime}\right) \hat{\mathbf{\Psi}}_{C}\left(\mathbf{z}_{2}\right) \hat{\mathbf{\Psi}}_{C}\left(\mathbf{z}_{1}\right)\left|\Psi^{(A B C)}(t)\right\rangle\right\}= \\
& =\sum_{k, k^{\prime \prime}, s^{\prime \prime}, l^{\prime \prime}, q, q^{\prime \prime}=1}^{M_{A}, M_{C}} \rho_{k k^{\prime \prime} s^{\prime \prime} l^{\prime \prime} q q^{\prime \prime}}^{(A C C)}(t) \phi_{k}^{*}\left(\mathbf{x}_{1}^{\prime}, t\right) \phi_{q}\left(\mathbf{x}_{1}, t\right) \chi_{k^{\prime \prime}}^{*}\left(\mathbf{z}_{1}^{\prime}, t\right) \chi_{s^{\prime \prime}}^{*}\left(\mathbf{z}_{2}^{\prime}, t\right) \chi_{l^{\prime \prime}}\left(\mathbf{z}_{2}, t\right) \chi_{q^{\prime \prime}}\left(\mathbf{z}_{1}, t\right),
\end{aligned}
$$

where

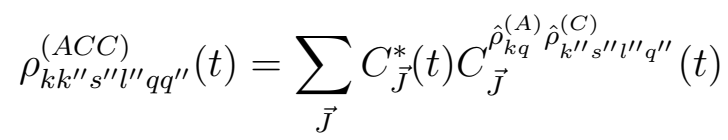

are its matrix elements in the orbital basis.

$$
\begin{aligned}
& \rho^{(B B C)}\left(\mathbf{y}_{1}, \mathbf{y}_{2}, \mathbf{z}_{1} \mid \mathbf{y}_{1}^{\prime}, \mathbf{y}_{2}^{\prime}, \mathbf{z}_{1}^{\prime} ; t\right)= \\
& =N_{B}\left(N_{B}-1\right) N_{C} \int d \mathbf{x}_{1} \cdots d \mathbf{x}_{N_{A}} d \mathbf{y}_{3} \cdots d \mathbf{y}_{N_{B}} d \mathbf{z}_{2} \cdots d \mathbf{z}_{N_{C}} \times \\
& \times \Psi^{(A B C)^{*}}\left(\mathbf{x}_{1}, \mathbf{x}_{2}, \ldots, \mathbf{x}_{N_{A}}, \mathbf{y}_{1}^{\prime}, \mathbf{y}_{2}^{\prime}, \ldots, \mathbf{y}_{N_{B}}, \mathbf{z}_{1}^{\prime}, \mathbf{z}_{2}, \ldots, \mathbf{z}_{N_{C}} ; t\right) \times \\
& \times \Psi^{(A B C)}\left(\mathbf{x}_{1}, \mathbf{x}_{2}, \ldots, \mathbf{x}_{N_{A}}, \mathbf{y}_{1}, \mathbf{y}_{2}, \ldots, \mathbf{y}_{N_{B}}, \mathbf{z}_{1}, \mathbf{z}_{2}, \ldots, \mathbf{z}_{N_{C}} ; t\right)= \\
& =\left\langle\Psi^{(A B C)}(t)\right|\left\{\hat{\mathbf{\Psi}}_{B}^{\dagger}\left(\mathbf{y}_{1}^{\prime}\right) \hat{\mathbf{\Psi}}_{B}^{\dagger}\left(\mathbf{y}_{2}^{\prime}\right) \hat{\mathbf{\Psi}}_{B}\left(\mathbf{y}_{2}\right) \hat{\mathbf{\Psi}}_{B}\left(\mathbf{y}_{1}\right) \hat{\mathbf{\Psi}}_{C}^{\dagger}\left(\mathbf{z}_{1}^{\prime}\right) \hat{\mathbf{\Psi}}_{C}\left(\mathbf{z}_{1}\right)\left|\Psi^{(A B C)}(t)\right\rangle\right\}= \\
& =\sum_{k^{\prime}, k^{\prime \prime}, s^{\prime}, l^{\prime}, q^{\prime}, q^{\prime \prime}=1}^{M_{B}, M_{C}} \rho_{k^{\prime} k^{\prime \prime} s^{\prime} l^{\prime} q^{\prime} q^{\prime \prime}}^{(B B C)}(t) \psi_{k^{\prime}}^{*}\left(\mathbf{y}_{1}^{\prime}, t\right) \psi_{s^{\prime}}^{*}\left(\mathbf{y}_{2}^{\prime}, t\right) \psi_{l^{\prime}}\left(\mathbf{y}_{2}, t\right) \phi_{q^{\prime}}\left(\mathbf{y}_{1}, t\right) \chi_{k^{\prime \prime}}^{*}\left(\mathbf{z}_{1}^{\prime}, t\right) \chi_{q^{\prime \prime}}\left(\mathbf{z}_{1}, t\right),
\end{aligned}
$$

where

$$
\rho_{k^{\prime} k^{\prime \prime} s^{\prime} l^{\prime} q^{\prime} q^{\prime \prime}}^{(B B C)}(t)=\sum_{\vec{J}} C_{\vec{J}}^{*}(t) C_{\vec{J}}^{\hat{\rho}_{k^{\prime} s^{\prime} l^{\prime} q^{\prime}}^{(B)} \hat{\rho}_{k^{\prime \prime} q^{\prime \prime}}^{(C)}}(t)
$$

are its matrix elements in the orbital basis. 


$$
\begin{aligned}
& \rho^{(B C C)}\left(\mathbf{y}_{1}, \mathbf{z}_{1}, \mathbf{z}_{2} \mid \mathbf{y}_{1}^{\prime}, \mathbf{z}_{1}^{\prime}, \mathbf{z}_{2}^{\prime} ; t\right)= \\
& =N_{B} N_{C}\left(N_{C}-1\right) \int d \mathbf{x}_{1} \cdots d \mathbf{x}_{N_{A}} d \mathbf{y}_{2} \cdots d \mathbf{y}_{N_{B}} d \mathbf{z}_{3} \cdots d \mathbf{z}_{N_{C}} \times \\
& \times \Psi^{(A B C)^{*}}\left(\mathbf{x}_{1}, \mathbf{x}_{2}, \ldots, \mathbf{x}_{N_{A}}, \mathbf{y}_{1}^{\prime}, \mathbf{y}_{2}, \ldots, \mathbf{y}_{N_{B}}, \mathbf{z}_{1}^{\prime}, \mathbf{z}_{2}^{\prime}, \ldots, \mathbf{z}_{N_{C}} ; t\right) \times \\
& \times \Psi^{(A B C)}\left(\mathbf{x}_{1}, \mathbf{x}_{2}, \ldots, \mathbf{x}_{N_{A}}, \mathbf{y}_{1}, \mathbf{y}_{2}, \ldots, \mathbf{y}_{N_{B}}, \mathbf{z}_{1}, \mathbf{z}_{2}, \ldots, \mathbf{z}_{N_{C}} ; t\right)= \\
& =\left\langle\Psi^{(A B C)}(t)\right|\left\{\hat{\Psi}_{B}^{\dagger}\left(\mathbf{y}_{1}^{\prime}\right) \hat{\mathbf{\Psi}}_{B}\left(\mathbf{y}_{1}\right) \hat{\mathbf{\Psi}}_{C}^{\dagger}\left(\mathbf{z}_{1}^{\prime}\right) \hat{\mathbf{\Psi}}_{C}^{\dagger}\left(\mathbf{z}_{2}^{\prime}\right) \hat{\mathbf{\Psi}}_{C}\left(\mathbf{z}_{2}\right) \hat{\mathbf{\Psi}}_{C}\left(\mathbf{z}_{1}\right)\left|\Psi^{(A B C)}(t)\right\rangle\right\}= \\
& =\sum_{k^{\prime}, k^{\prime \prime}, s^{\prime \prime}, l^{\prime \prime}, q^{\prime}, q^{\prime \prime}=1}^{M_{B}, M_{C}} \rho_{k^{\prime} k^{\prime \prime} s^{\prime \prime} l^{\prime \prime} q^{\prime} q^{\prime \prime}}^{(B C C)}(t) \psi_{k^{\prime}}^{*}\left(\mathbf{y}_{1}^{\prime}, t\right) \psi_{q^{\prime}}\left(\mathbf{y}_{1}, t\right) \chi_{k^{\prime \prime}}^{*}\left(\mathbf{z}_{1}^{\prime}, t\right) \chi_{s^{\prime \prime}}^{*}\left(\mathbf{z}_{2}^{\prime}, t\right) \chi_{l^{\prime \prime}}\left(\mathbf{z}_{2}, t\right) \chi_{q^{\prime \prime}}\left(\mathbf{z}_{1}, t\right),
\end{aligned}
$$

where

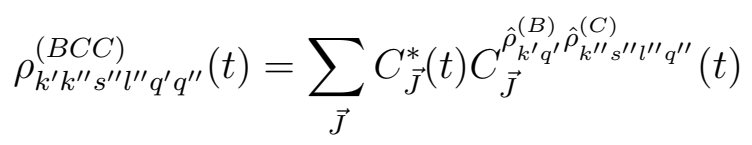

are its matrix elements in the orbital basis.

Finally, there is a single reduced three-body density matrix which is associated with the three-body interaction of three distinct particles.

$$
\begin{aligned}
& \rho^{(A B C)}\left(\mathbf{x}_{1}, \mathbf{y}_{1}, \mathbf{z}_{1} \mid \mathbf{x}_{1}^{\prime}, \mathbf{y}_{1}^{\prime}, \mathbf{z}_{1}^{\prime} ; t\right)= \\
& =N_{A} N_{B} N_{C} \int d \mathbf{x}_{2} \cdots d \mathbf{x}_{N_{A}} d \mathbf{y}_{2} \cdots d \mathbf{y}_{N_{B}} d \mathbf{z}_{2} \cdots d \mathbf{z}_{N_{C}} \times \\
& \times \Psi^{(A B C)^{*}}\left(\mathbf{x}_{1}^{\prime}, \mathbf{x}_{2}, \ldots, \mathbf{x}_{N_{A}}, \mathbf{y}_{1}^{\prime}, \mathbf{y}_{2}, \ldots, \mathbf{y}_{N_{B}}, \mathbf{z}_{1}^{\prime}, \mathbf{z}_{2}, \ldots, \mathbf{z}_{N_{C}} ; t\right) \times \\
& \times \Psi^{(A B C)}\left(\mathbf{x}_{1}, \mathbf{x}_{2}, \ldots, \mathbf{x}_{N_{A}}, \mathbf{y}_{1}, \mathbf{y}_{2}, \ldots, \mathbf{y}_{N_{B}}, \mathbf{z}_{1}, \mathbf{z}_{2}, \ldots, \mathbf{z}_{N_{C}} ; t\right)= \\
& =\left\langle\Psi^{(A B C)}(t)\right|\left\{\hat{\mathbf{\Psi}}_{A}^{\dagger}\left(\mathbf{x}_{1}^{\prime}\right) \hat{\mathbf{\Psi}}_{A}\left(\mathbf{x}_{1}\right) \hat{\mathbf{\Psi}}_{B}^{\dagger}\left(\mathbf{y}_{1}^{\prime}\right) \hat{\mathbf{\Psi}}_{B}\left(\mathbf{y}_{1}\right) \hat{\mathbf{\Psi}}_{C}^{\dagger}\left(\mathbf{z}_{2}^{\prime}\right) \hat{\mathbf{\Psi}}_{C}\left(\mathbf{z}_{1}\right)\left|\Psi^{(A B C)}(t)\right\rangle\right\}= \\
& =\sum_{M_{A}, M_{B}, M_{C}}^{(A B C)} \rho_{k k^{\prime} k^{\prime \prime} q q^{\prime} q^{\prime \prime}}^{(t), k^{\prime \prime}, q, q^{\prime}, q^{\prime \prime}=1}(t) \phi_{k}^{*}\left(\mathbf{x}_{1}^{\prime}, t\right) \phi_{q}\left(\mathbf{x}_{1}, t\right) \psi_{k^{\prime}}^{*}\left(\mathbf{y}_{1}^{\prime}, t\right) \psi_{q^{\prime}}\left(\mathbf{y}_{1}, t\right), \chi_{k}^{*}\left(\mathbf{y}_{1}^{\prime}, t\right) \chi_{q}\left(\mathbf{y}_{1}, t\right)
\end{aligned}
$$

where

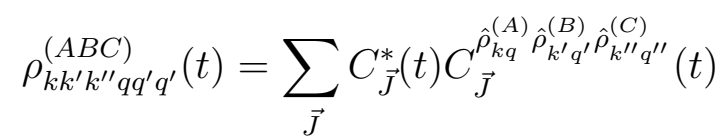

are its matrix elements in the orbital basis. 
Appendix C: Further details of the derivation of the equations-of-motion for mixtures of three kinds of identical particles

The derivation of the equations-of-motion for the orbitals (51) starts from expressing the

expectation value of $\hat{H}^{(A B C)}$ with respect to the many-particle wave-function $\left|\Psi^{(A B C)}\right\rangle$ in a form which depends explicitly on the various integrals with respect to the orbitals. Thus we 
have:

$$
\begin{aligned}
& \left\langle\Psi^{(A B C)}\left|\hat{H}^{(A B C)}-i \frac{\partial}{\partial t}\right| \Psi^{(A B C)}\right\rangle=\sum_{k, q=1}^{M_{A}} \rho_{k q}^{(A)}\left[h_{k q}^{(A)}-\left\{i \frac{\partial}{\partial t}^{(A)}\right\}_{k q}\right]+ \\
& +\frac{1}{2} \sum_{k, s, l, q=1}^{M_{A}} \rho_{k s l q}^{(A)} W_{k s q l}^{(A)}+\frac{1}{6} \sum_{k, s, p, r, l, q=1}^{M_{A}} \rho_{k s p r l q}^{(A)} U_{k s p q l r}^{(A)}+ \\
& +\sum_{k^{\prime}, q^{\prime}=1}^{M_{B}} \rho_{k^{\prime} q^{\prime}}^{(B)}\left[h_{k^{\prime} q^{\prime}}^{(B)}-\left\{i \frac{\partial}{\partial t}^{(B)}\right\}_{k^{\prime} q^{\prime}}\right]+ \\
& +\frac{1}{2} \sum_{k^{\prime}, s^{\prime}, l^{\prime}, q^{\prime}=1}^{M_{B}} \rho_{k^{\prime} s^{\prime} l^{\prime} q^{\prime}}^{(B)} W_{k^{\prime} s^{\prime} q^{\prime} l^{\prime}}^{(B)}+\frac{1}{6} \sum_{k^{\prime}, s^{\prime}, p^{\prime}, r^{\prime}, l^{\prime}, q^{\prime}=1}^{M_{B}} \rho_{k^{\prime} s^{\prime} p^{\prime} r^{\prime} l^{\prime} q^{\prime}}^{(B)} U_{k^{\prime} s^{\prime} p^{\prime} q^{\prime} l^{\prime} r^{\prime}}^{(B)}+ \\
& +\sum_{k^{\prime \prime}, q^{\prime \prime}=1}^{M_{C}} \rho_{k^{\prime \prime} q^{\prime \prime}}^{(C)}\left[h_{k^{\prime \prime} q^{\prime \prime}}^{(C)}-\left\{i \frac{\partial}{\partial t}^{(C)}\right\}_{k^{\prime \prime} q^{\prime \prime}}\right]+ \\
& +\frac{1}{2} \sum_{k^{\prime \prime}, s^{\prime \prime}, l^{\prime \prime}, q^{\prime \prime}=1}^{M_{C}} \rho_{k^{\prime \prime} s^{\prime \prime} l^{\prime \prime} q^{\prime \prime}}^{(C)} W_{k^{\prime \prime} s^{\prime \prime} q^{\prime \prime} l^{\prime \prime}}^{(C)}+ \\
& +\frac{1}{6} \sum_{k^{\prime \prime}, s^{\prime \prime}, p^{\prime \prime}, r^{\prime \prime}, l^{\prime \prime}, q^{\prime \prime}=1}^{M_{C}} \rho_{k^{\prime \prime} s^{\prime \prime} p^{\prime \prime} r^{\prime \prime} l^{\prime \prime} q^{\prime \prime}}^{(C)} U_{k^{\prime \prime} s^{\prime \prime} p^{\prime \prime} q^{\prime \prime} l^{\prime \prime} r^{\prime \prime}}^{(C)}+ \\
& +\sum_{k, k^{\prime}, q, q^{\prime}=1}^{M_{A}, M_{B}} \rho_{k k^{\prime} q q^{\prime}}^{(A B)} W_{k k^{\prime} q q^{\prime}}^{(A B)}+\sum_{k, k^{\prime \prime}, q, q^{\prime \prime}=1}^{M_{A}, M_{C}} \rho_{k k^{\prime \prime} q q^{\prime \prime}}^{(A C)} W_{k k^{\prime \prime} q q^{\prime \prime}}^{(A C)}+ \\
& +\sum_{k^{\prime}, k^{\prime \prime}, q^{\prime}, q^{\prime \prime}=1}^{M_{B}, M_{C}} \rho_{k^{\prime} k^{\prime \prime} q^{\prime} q^{\prime \prime}}^{(B C)} W_{k^{\prime} k^{\prime \prime} q^{\prime} q^{\prime \prime}}^{(B C)}+ \\
& +\frac{1}{2} \sum_{k, k^{\prime}, s, q, q^{\prime}, l=1}^{M_{A}, M_{B}} \rho_{k k^{\prime} s l q q^{\prime}}^{(A A B)} U_{k k^{\prime} s q q^{\prime} l}^{(A A B)}+\frac{1}{2} \sum_{k, k^{\prime}, s^{\prime}, q, q^{\prime}, l^{\prime}=1}^{M_{A}, M_{B}} \rho_{k k^{\prime} s^{\prime} l^{\prime} q q^{\prime}}^{(A B B)} U_{k k^{\prime} s^{\prime} q q^{\prime} l^{\prime}}^{(A B B)}+ \\
& +\frac{1}{2} \sum_{k, k^{\prime \prime}, s, q, q^{\prime \prime}, l=1}^{M_{A}, M_{C}} \rho_{k k^{\prime \prime} s l q q^{\prime \prime}}^{(A A C)} U_{k k^{\prime \prime} s q q^{\prime \prime l} l}^{(A A C)}+\frac{1}{2} \sum_{k, k^{\prime \prime}, s^{\prime \prime}, q, q^{\prime \prime}, l^{\prime \prime}=1}^{M_{A}, M_{C}} \rho_{k k^{\prime \prime} s^{\prime \prime} l^{\prime \prime} q q^{\prime \prime}}^{(A C C)} U_{k k^{\prime \prime} s^{\prime \prime} q q^{\prime \prime} l^{\prime \prime}}^{(A C C)}+ \\
& +\frac{1}{2} \sum_{k^{\prime}, k^{\prime \prime}, s^{\prime}, q^{\prime}, q^{\prime \prime}, l^{\prime}=1}^{M_{B}, M_{C}} \rho_{k^{\prime} k^{\prime \prime} s^{\prime} l^{\prime} q^{\prime} q^{\prime \prime}}^{(B B C)} U_{k^{\prime} k^{\prime \prime} s^{\prime} q^{\prime} q^{\prime \prime} l^{\prime}}^{(B B C)}+\frac{1}{2} \sum_{k^{\prime}, k^{\prime \prime}, s^{\prime \prime}, q^{\prime}, q^{\prime \prime}, l^{\prime \prime}=1}^{M_{B}, M_{C}} \rho_{k^{\prime} k^{\prime \prime} s^{\prime \prime} l^{\prime \prime} q^{\prime} q^{\prime \prime}}^{(B C C)} U_{k^{\prime} k^{\prime \prime} s^{\prime \prime} q^{\prime} q^{\prime \prime} l^{\prime \prime}}^{(B C C)}+ \\
& +\sum_{k, k^{\prime}, k^{\prime \prime}, q, q^{\prime}, q^{\prime \prime}=1}^{M_{A}, M_{B}, M_{C}} \rho_{k k^{\prime} k^{\prime \prime} q q^{\prime} q^{\prime \prime}}^{(A B C)} U_{k k^{\prime} k^{\prime \prime} q q^{\prime} q^{\prime \prime}}^{(A B C)}-\sum_{\{\vec{J}\}} i C_{\vec{J}}^{*}(t) \dot{C}_{\vec{J}}(t) .
\end{aligned}
$$

The expectation values of the various density operators appearing in (C1) have been prescribed in Appendix B.

The matrix elements in (C1) of the $A$ and correspondingly of the $B$ and $C$ single-species terms with respect to the orbitals have been discussed in section II A, see Eq. (4). The 
matrix elements arising from two-body inter-species interactions are listed for completeness below:

$$
\begin{aligned}
W_{k k^{\prime} q q^{\prime}}^{(A B)} & =\iint \phi_{k}^{*}(\mathbf{x}, t) \psi_{k^{\prime}}^{*}(\mathbf{y}, t) \hat{W}^{(A B)}(\mathbf{x}, \mathbf{y}) \phi_{q}(\mathbf{x}, t) \psi_{q^{\prime}}(\mathbf{y}, t) d \mathbf{x} d \mathbf{y} \\
W_{k k^{\prime \prime} q q^{\prime \prime}}^{(A C)} & =\iint \phi_{k}^{*}(\mathbf{x}, t) \chi_{k^{\prime \prime}}^{*}(\mathbf{z}, t) \hat{W}^{(A C)}(\mathbf{x}, \mathbf{z}) \phi_{q}(\mathbf{x}, t) \chi_{q^{\prime \prime}}(\mathbf{z}, t) d \mathbf{x} d \mathbf{z} \\
W_{k^{\prime} k^{\prime \prime} q^{\prime} q^{\prime \prime}}^{(B C)} & =\iint \psi_{k^{\prime}}^{*}(\mathbf{y}, t) \chi_{k^{\prime \prime}}^{*}(\mathbf{z}, t) \hat{W}^{(B C)}(\mathbf{y}, \mathbf{z}) \psi_{q^{\prime}}(\mathbf{y}, t) \chi_{q^{\prime \prime}}(\mathbf{z}, t) d \mathbf{y} d \mathbf{z},
\end{aligned}
$$

and the matrix elements arising from three-body inter-species interactions read as follows:

$$
\begin{aligned}
& U_{k k^{\prime} s q q^{\prime} l}^{(A A B)}= \\
& =\iiint \phi_{k}^{*}(\mathbf{x}, t) \phi_{s}^{*}\left(\mathbf{x}^{\prime}, t\right) \psi_{k^{\prime}}^{*}(\mathbf{y}, t) \hat{U}^{(A A B)}\left(\mathbf{x}, \mathbf{x}^{\prime}, \mathbf{y}\right) \phi_{q}(\mathbf{x}, t) \phi_{l}\left(\mathbf{x}^{\prime}, t\right) \psi_{q^{\prime}}(\mathbf{y}, t) d \mathbf{x} d \mathbf{x}^{\prime} d \mathbf{y}, \\
& U_{k k^{\prime} s^{\prime} q q^{\prime} l^{\prime}}^{(A B B)}= \\
& =\iiint \phi_{k}^{*}(\mathbf{x}, t) \psi_{k^{\prime}}^{*}(\mathbf{y}, t) \psi_{s^{\prime}}^{*}\left(\mathbf{y}^{\prime}, t\right) \hat{U}^{(A B B)}\left(\mathbf{x}, \mathbf{y}, \mathbf{y}^{\prime}\right) \phi_{q}(\mathbf{x}, t) \psi_{q^{\prime}}(\mathbf{y}, t) \psi_{l^{\prime}}\left(\mathbf{y}^{\prime}, t\right) d \mathbf{x} d \mathbf{y} d \mathbf{y}^{\prime}, \\
& U_{k k^{\prime \prime} s q q^{\prime \prime l}}^{(A A C)}= \\
& =\iiint \phi_{k}^{*}(\mathbf{x}, t) \phi_{s}^{*}\left(\mathbf{x}^{\prime}, t\right) \chi_{k^{\prime \prime}}^{*}(\mathbf{z}, t) \hat{U}^{(A A C)}\left(\mathbf{x}, \mathbf{x}^{\prime}, \mathbf{z}\right) \phi_{q}(\mathbf{x}, t) \phi_{l}\left(\mathbf{x}^{\prime}, t\right) \chi_{q^{\prime \prime}}(\mathbf{z}, t) d \mathbf{x} d \mathbf{x}^{\prime} d \mathbf{z}, \\
& U_{k k^{\prime \prime} s^{\prime \prime} q q^{\prime \prime} l^{\prime \prime}}^{(A C C)}= \\
& =\iiint \phi_{k}^{*}(\mathbf{x}, t) \chi_{k^{\prime \prime}}^{*}(\mathbf{z}, t) \chi_{s^{\prime \prime}}^{*}\left(\mathbf{z}^{\prime}, t\right) \hat{U}^{(A C C)}\left(\mathbf{x}, \mathbf{z}, \mathbf{z}^{\prime}\right) \phi_{q}(\mathbf{x}, t) \chi_{q^{\prime \prime}}(\mathbf{z}, t) \chi_{l^{\prime \prime}}\left(\mathbf{z}^{\prime}, t\right) d \mathbf{x} d \mathbf{z} d \mathbf{z}^{\prime}, \\
& U_{k^{\prime} k^{\prime \prime} s^{\prime} q^{\prime} q^{\prime \prime} l^{\prime}}^{(B B C)}= \\
& =\iiint \psi_{k^{\prime}}^{*}(\mathbf{y}, t) \psi_{s^{\prime}}^{*}\left(\mathbf{y}^{\prime}, t\right) \chi_{k^{\prime \prime}}^{*}(\mathbf{z}, t) \hat{U}^{(B B C)}\left(\mathbf{y}, \mathbf{y}^{\prime}, \mathbf{z}\right) \psi_{q^{\prime}}(\mathbf{y}, t) \psi_{l^{\prime}}\left(\mathbf{y}^{\prime}, t\right) \chi_{q^{\prime \prime}}(\mathbf{z}, t) d \mathbf{y} d \mathbf{y}^{\prime} d \mathbf{z} \\
& U_{k^{\prime} k^{\prime \prime} s^{\prime \prime} q^{\prime} q^{\prime \prime} l^{\prime \prime}}^{(B C C)}= \\
& =\iiint \psi_{k^{\prime}}^{*}(\mathbf{y}, t) \chi_{k^{\prime \prime}}^{*}(\mathbf{z}, t) \chi_{s^{\prime \prime}}^{*}\left(\mathbf{z}^{\prime}, t\right) \hat{U}^{(B C C)}\left(\mathbf{y}, \mathbf{z}, \mathbf{z}^{\prime}\right) \psi_{q^{\prime}}(\mathbf{y}, t) \chi_{q^{\prime \prime}}(\mathbf{z}, t) \chi_{l^{\prime \prime}}\left(\mathbf{z}^{\prime}, t\right) d \mathbf{y} d \mathbf{z} d \mathbf{z}^{\prime}, \\
& U_{k k^{\prime} k^{\prime \prime} q q^{\prime} q^{\prime \prime}}^{(A B C)}= \\
& =\iiint \phi_{k}^{*}(\mathbf{x}, t) \psi_{k^{\prime}}^{*}(\mathbf{y}, t) \chi_{k^{\prime \prime}}^{*}(\mathbf{z}, t) \hat{U}^{(A B C)}(\mathbf{x}, \mathbf{y}, \mathbf{z}) \phi_{q}(\mathbf{x}, t) \psi_{q^{\prime}}(\mathbf{y}, t) \chi_{q^{\prime \prime}}(\mathbf{z}, t) d \mathbf{x} d \mathbf{y} d \mathbf{z} .
\end{aligned}
$$

Performing the variation of the integrals (C2) with respect to the orbitals $\left\{\phi_{k}(\mathbf{x}, t)\right\}$, $\left\{\psi_{k^{\prime}}(\mathbf{y}, t)\right\}$ and $\left\{\chi_{k^{\prime \prime}}(\mathbf{z}, t)\right\}$, we find six types of inter-species one-body potentials emerging 
from two-body interactions:

$$
\begin{aligned}
\hat{W}_{k^{\prime} q^{\prime}}^{(A B)}(\mathbf{x}, t) & =\int \psi_{k^{\prime}}^{*}(\mathbf{y}, t) \hat{W}^{(A B)}(\mathbf{x}, \mathbf{y}) \psi_{q^{\prime}}(\mathbf{y}, t) d \mathbf{y}, \\
\hat{W}_{k q}^{(B A)}(\mathbf{y}, t) & =\int \phi_{k}^{*}(\mathbf{x}, t) \hat{W}^{(A B)}(\mathbf{x}, \mathbf{y}) \phi_{q}(\mathbf{x}, t) d \mathbf{x} \\
\hat{W}_{k^{\prime \prime} q^{\prime \prime}}^{(A C)}(\mathbf{x}, t) & =\int \chi_{k^{\prime \prime}}^{*}(\mathbf{z}, t) \hat{W}^{(A C)}(\mathbf{x}, \mathbf{z}) \chi_{q^{\prime \prime}}(\mathbf{z}, t) d \mathbf{z}, \\
\hat{W}_{k q}^{(C A)}(\mathbf{z}, t) & =\int \phi_{k}^{*}(\mathbf{x}, t) \hat{W}^{(A C)}(\mathbf{x}, \mathbf{z}) \phi_{q}(\mathbf{x}, t) d \mathbf{x}, \\
\hat{W}_{k^{\prime \prime} q^{\prime \prime}}^{(B C)}(\mathbf{y}, t) & =\int \chi_{k^{\prime \prime}}^{*}(\mathbf{z}, t) \hat{W}^{(B C)}(\mathbf{y}, \mathbf{z}) \chi_{q^{\prime \prime}}(\mathbf{z}, t) d \mathbf{z}, \\
\hat{W}_{k^{\prime} q^{\prime}}^{(C B)}(\mathbf{z}, t) & =\int \psi_{k^{\prime}}^{*}(\mathbf{y}, t) \hat{W}^{(B C)}(\mathbf{y}, \mathbf{z}) \psi_{q^{\prime}}(\mathbf{y}, t) d \mathbf{y} .
\end{aligned}
$$

Making the variation of the integrals (C3) with respect to the orbitals, we arrive at fifteen types of inter-species one-body potentials resulting from three-body interactions: 


$$
\begin{aligned}
& \hat{U}_{s k^{\prime} l q^{\prime}}^{(A A B)}(\mathbf{x}, t)=\iint \phi_{s}^{*}\left(\mathbf{x}^{\prime}, t\right) \psi_{k^{\prime}}^{*}(\mathbf{y}, t) \hat{U}^{(A A B)}\left(\mathbf{x}, \mathbf{x}^{\prime}, \mathbf{y}\right) \phi_{l}\left(\mathbf{x}^{\prime}, t\right) \psi_{q^{\prime}}(\mathbf{y}, t) d \mathbf{x}^{\prime} d \mathbf{y} \\
& \hat{U}_{k s q l}^{(B A A)}(\mathbf{y}, t)=\iint \phi_{k}^{*}(\mathbf{x}, t) \phi_{s}^{*}\left(\mathbf{x}^{\prime}, t\right) \hat{U}^{(A A B)}\left(\mathbf{x}, \mathbf{x}^{\prime}, \mathbf{y}\right) \phi_{q}(\mathbf{x}, t) \phi_{l}\left(\mathbf{x}^{\prime}, t\right) d \mathbf{x} d \mathbf{x}^{\prime}, \\
& \hat{U}_{k^{\prime} s^{\prime} q^{\prime} l^{\prime}}^{(A B B)}(\mathbf{x}, t)=\iint \psi_{k^{\prime}}^{*}(\mathbf{y}, t) \psi_{s^{\prime}}^{*}\left(\mathbf{y}^{\prime}, t\right) \hat{U}^{(A B B)}\left(\mathbf{x}, \mathbf{y}, \mathbf{y}^{\prime}\right) \psi_{q^{\prime}}(\mathbf{y}, t) \psi_{l^{\prime}}\left(\mathbf{y}^{\prime}, t\right) d \mathbf{y} d \mathbf{y}^{\prime}, \\
& \hat{U}_{k s^{\prime} q l^{\prime}}^{(B A B)}(\mathbf{y}, t)=\iint \phi_{k}^{*}(\mathbf{x}, t) \psi_{s^{\prime}}^{*}\left(\mathbf{y}^{\prime}, t\right) \hat{U}^{(A B B)}\left(\mathbf{x}, \mathbf{y}, \mathbf{y}^{\prime}\right) \phi_{q}(\mathbf{x}, t) \psi_{l^{\prime}}\left(\mathbf{y}^{\prime}, t\right) d \mathbf{x} d \mathbf{y}^{\prime}, \\
& \hat{U}_{s k^{\prime \prime} l q^{\prime \prime}}^{(A A C)}(\mathbf{x}, t)=\iint \phi_{s}^{*}\left(\mathbf{x}^{\prime}, t\right) \chi_{k^{\prime \prime}}^{*}(\mathbf{z}, t) \hat{U}^{(A A C)}\left(\mathbf{x}, \mathbf{x}^{\prime}, \mathbf{z}\right) \phi_{l}\left(\mathbf{x}^{\prime}, t\right) \chi_{q^{\prime \prime}}(\mathbf{z}, t) d \mathbf{x}^{\prime} d \mathbf{z} \\
& \hat{U}_{k s q l}^{(C A A)}(\mathbf{z}, t)=\iint \phi_{k}^{*}(\mathbf{x}, t) \phi_{s}^{*}\left(\mathbf{x}^{\prime}, t\right) \hat{U}^{(A A C)}\left(\mathbf{x}, \mathbf{x}^{\prime}, \mathbf{z}\right) \phi_{q}(\mathbf{x}, t) \phi_{l}\left(\mathbf{x}^{\prime}, t\right) d \mathbf{x} d \mathbf{x}^{\prime}, \\
& \hat{U}_{k^{\prime \prime} s^{\prime \prime} q^{\prime \prime} l^{\prime \prime}}^{(A C C)}(\mathbf{x}, t)=\iint \chi_{k^{\prime \prime}}^{*}(\mathbf{z}, t) \chi_{s^{\prime \prime}}^{*}\left(\mathbf{z}^{\prime}, t\right) \hat{U}^{(A C C)}\left(\mathbf{x}, \mathbf{z}, \mathbf{z}^{\prime}\right) \chi_{q^{\prime \prime}}(\mathbf{z}, t) \chi_{l^{\prime \prime}}\left(\mathbf{z}^{\prime}, t\right) d \mathbf{z} d \mathbf{z}^{\prime}, \\
& \hat{U}_{k s^{\prime \prime} q l^{\prime \prime}}^{(C A C)}(\mathbf{z}, t)=\iint \phi_{k}^{*}(\mathbf{x}, t) \chi_{s^{\prime \prime}}^{*}\left(\mathbf{z}^{\prime}, t\right) \hat{U}^{(A C C)}\left(\mathbf{x}, \mathbf{z}, \mathbf{z}^{\prime}\right) \phi_{q}(\mathbf{x}, t) \chi_{l^{\prime \prime}}\left(\mathbf{z}^{\prime}, t\right) d \mathbf{x} d \mathbf{z}^{\prime}, \\
& \hat{U}_{s^{\prime} k^{\prime \prime} l^{\prime} q^{\prime \prime}}^{(B B C)}(\mathbf{y}, t)=\iint \psi_{s^{\prime}}^{*}\left(\mathbf{y}^{\prime}, t\right) \chi_{k^{\prime \prime}}^{*}(\mathbf{z}, t) \hat{U}^{(B B C)}\left(\mathbf{y}, \mathbf{y}^{\prime}, \mathbf{z}\right) \psi_{l^{\prime}}\left(\mathbf{y}^{\prime}, t\right) \chi_{q^{\prime \prime}}(\mathbf{z}, t) d \mathbf{y}^{\prime} d \mathbf{z}, \\
& \hat{U}_{k^{\prime} s^{\prime} q^{\prime} l^{\prime}}^{(C B B)}(\mathbf{z}, t)=\iint \psi_{k^{\prime}}^{*}(\mathbf{y}, t) \psi_{s^{\prime}}^{*}\left(\mathbf{y}^{\prime}, t\right) \hat{U}^{(B B C)}\left(\mathbf{y}, \mathbf{y}^{\prime}, \mathbf{z}\right) \psi_{q^{\prime}}(\mathbf{y}, t) \psi_{l^{\prime}}\left(\mathbf{y}^{\prime}, t\right) d \mathbf{y} d \mathbf{y}^{\prime}, \\
& \hat{U}_{k^{\prime \prime} s^{\prime \prime} q^{\prime \prime} l^{\prime \prime}}^{(B C C)}(\mathbf{y}, t)=\iint \chi_{k^{\prime \prime}}^{*}(\mathbf{z}, t) \chi_{s^{\prime \prime}}^{*}\left(\mathbf{z}^{\prime}, t\right) \hat{U}^{(B C C)}\left(\mathbf{y}, \mathbf{z}, \mathbf{z}^{\prime}\right) \chi_{q^{\prime \prime}}(\mathbf{z}, t) \chi_{l^{\prime \prime}}\left(\mathbf{z}^{\prime}, t\right) d \mathbf{z} d \mathbf{z}^{\prime}, \\
& \hat{U}_{k^{\prime} s^{\prime \prime} q^{\prime} l^{\prime \prime}}^{(C B C)}(\mathbf{z}, t)=\iint \psi_{k^{\prime}}^{*}(\mathbf{y}, t) \chi_{s^{\prime \prime}}^{*}\left(\mathbf{z}^{\prime}, t\right) \hat{U}^{(B C C)}\left(\mathbf{y}, \mathbf{z}, \mathbf{z}^{\prime}\right) \psi_{q^{\prime}}(\mathbf{y}, t) \chi_{l^{\prime \prime}}\left(\mathbf{z}^{\prime}, t\right) d \mathbf{y} d \mathbf{z}^{\prime}, \\
& \hat{U}_{k^{\prime} k^{\prime \prime} q^{\prime} q^{\prime \prime}}^{(A B C)}(\mathbf{x}, t)=\iint \psi_{k^{\prime}}^{*}(\mathbf{y}, t) \chi_{k^{\prime \prime}}^{*}(\mathbf{z}, t) \hat{U}^{(A B C)}(\mathbf{x}, \mathbf{y}, \mathbf{z}) \psi_{q^{\prime}}(\mathbf{y}, t) \chi_{q^{\prime \prime}}(\mathbf{z}, t) d \mathbf{y} d \mathbf{z}, \\
& \hat{U}_{k k^{\prime \prime} q q^{\prime \prime}}^{(B A C)}(\mathbf{y}, t)=\iint \phi_{k}^{*}(\mathbf{x}, t) \chi_{k^{\prime \prime}}^{*}(\mathbf{z}, t) \hat{U}^{(A B C)}(\mathbf{x}, \mathbf{y}, \mathbf{z}) \phi_{q}(\mathbf{x}, t) \chi_{q^{\prime \prime}}(\mathbf{z}, t) d \mathbf{x} d \mathbf{z}, \\
& \hat{U}_{k k^{\prime} q q^{\prime}}^{(C A B)}(\mathbf{z}, t)=\iint \phi_{k}^{*}(\mathbf{x}, t) \psi_{k^{\prime}}^{*}(\mathbf{y}, t) \hat{U}^{(A B C)}(\mathbf{x}, \mathbf{y}, \mathbf{z}) \phi_{q}(\mathbf{x}, t) \psi_{q^{\prime}}(\mathbf{y}, t) d \mathbf{x} d \mathbf{y} .
\end{aligned}
$$

All one-body potentials in (C4) and (C5) are local (for spin-independent interactions), timedependent potentials.

To arrive at the final form of the equations-of-motion (51), we define the auxiliary one- 
body operators for the $A$-species' particles:

$$
\begin{aligned}
& \left\{\rho_{2} \hat{W}\right\}_{k q}^{(A)} \equiv \sum_{s, l=1}^{M_{A}} \rho_{k s l q}^{(A)} \hat{W}_{s l}^{(A)}+\sum_{k^{\prime}, q^{\prime}=1}^{M_{B}} \rho_{k k^{\prime} q q^{\prime}}^{(A B)} \hat{W}_{k^{\prime} q^{\prime}}^{(A B)}+\sum_{k^{\prime \prime}, q^{\prime \prime}=1}^{M_{C}} \rho_{k k^{\prime \prime} q q^{\prime \prime}}^{(A C)} \hat{W}_{k^{\prime \prime} q^{\prime \prime}}^{(A C)}, \\
& \left\{\rho_{3} \hat{U}\right\}_{k q}^{(A)} \equiv \frac{1}{2} \sum_{s, p, l, r=1}^{M_{A}} \rho_{k s p r l q}^{(A)} \hat{U}_{s p l r}^{(A)}+\sum_{k^{\prime}, s, q^{\prime}, l=1}^{M_{A}, M_{B}} \rho_{k k^{\prime} s l q q^{\prime}}^{(A A B)} \hat{U}_{s k^{\prime} l q^{\prime}}^{(A A B)}+ \\
& +\sum_{k^{\prime}, s^{\prime}, q^{\prime}, l^{\prime}=1}^{M_{B}} \rho_{k k^{\prime} s^{\prime} l^{\prime} q q^{\prime}}^{(A B B)} \hat{U}_{k^{\prime} s^{\prime} q^{\prime} l^{\prime}}^{(A B B)}+\sum_{k^{\prime \prime}, s, q^{\prime \prime}, l=1}^{M_{A}, M_{C}} \rho_{k k^{\prime \prime} s l q q^{\prime \prime}}^{(A A C)} \hat{U}_{s k^{\prime \prime} l q^{\prime \prime}}^{(A A C)}+ \\
& +\sum_{k^{\prime \prime}, s^{\prime \prime}, q^{\prime \prime}, l^{\prime \prime}=1}^{M_{C}} \rho_{k k^{\prime \prime} s^{\prime \prime} l^{\prime \prime} q q^{\prime \prime}}^{(A C C)} \hat{U}_{k^{\prime \prime} s^{\prime \prime} q^{\prime \prime} l^{\prime \prime}}^{(A C C)}+\sum_{k^{\prime}, k^{\prime \prime}, q^{\prime}, q^{\prime \prime}=1}^{M_{B}, M_{C}} \rho_{k k^{\prime} k^{\prime \prime} q q^{\prime} q^{\prime \prime}}^{(A B C)} \hat{U}_{k^{\prime} k^{\prime \prime} q^{\prime} q^{\prime \prime},}^{(A B C)}
\end{aligned}
$$

for the $B$-species' particles:

$$
\begin{aligned}
& \left\{\rho_{2} \hat{W}\right\}_{k^{\prime} q^{\prime}}^{(B)} \equiv \sum_{s^{\prime}, l^{\prime}=1}^{M_{B}} \rho_{k^{\prime} s^{\prime} l^{\prime} q^{\prime}}^{(B)} \hat{W}_{s^{\prime} l^{\prime}}^{(B)}+\sum_{k, q=1}^{M_{A}} \rho_{k k^{\prime} q q^{\prime}}^{(A B)} \hat{W}_{k q}^{(B A)}+\sum_{k^{\prime \prime}, q^{\prime \prime}=1}^{M_{C}} \rho_{k^{\prime} k^{\prime \prime} q^{\prime} q^{\prime \prime}}^{(B C)} \hat{W}_{k^{\prime \prime} q^{\prime \prime}}^{(B C)}, \\
& \left\{\rho_{3} \hat{U}\right\}_{k^{\prime} q^{\prime}}^{(B)} \equiv \frac{1}{2} \sum_{s^{\prime}, p^{\prime}, r^{\prime}, l^{\prime}=1}^{M_{B}} \rho_{k^{\prime} s^{\prime} p^{\prime} r^{\prime} l^{\prime} q^{\prime}}^{(B)} \hat{U}_{s^{\prime} p^{\prime} l^{\prime} r^{\prime}}^{(B)}+\sum_{k, s, q, l=1}^{M_{A}} \rho_{k k^{\prime} s l q q^{\prime}}^{(A A B)} \hat{U}_{k s q l}^{(B A A)}+ \\
& +\sum_{k, s^{\prime}, q, l^{\prime}=1}^{M_{A}, M_{B}} \rho_{k k^{\prime} s^{\prime} l^{\prime} q q^{\prime}}^{(A B B)} \hat{U}_{k s^{\prime} q l^{\prime}}^{(B A B)}+\sum_{k^{\prime \prime}, s^{\prime}, q^{\prime \prime}, l^{\prime}=1}^{M_{B}, M_{C}} \rho_{k^{\prime} k^{\prime \prime} s^{\prime} l^{\prime} q^{\prime} q^{\prime \prime}}^{\left(B B U_{s^{\prime} k^{\prime \prime} l^{\prime} q^{\prime \prime}}^{(B B C)}+\right.} \\
& +\sum_{k^{\prime \prime}, s^{\prime \prime}, q^{\prime \prime}, l^{\prime \prime}=1}^{M_{C}} \rho_{k^{\prime} k^{\prime \prime} s^{\prime \prime} l^{\prime \prime} q^{\prime} q^{\prime \prime}}^{(B C C)} \hat{U}_{k^{\prime \prime} s^{\prime \prime} q^{\prime \prime} l^{\prime \prime}}^{(B C C)}+\sum_{k, k^{\prime \prime}, q, q^{\prime \prime}=1}^{M_{A}, M_{C}} \rho_{k k^{\prime} k^{\prime \prime} q q^{\prime} q^{\prime \prime}}^{(A B C)} \hat{U}_{k k^{\prime \prime} q q^{\prime \prime}}^{(B A C)},
\end{aligned}
$$

and for the $C$-species' particles:

$$
\begin{aligned}
& \left\{\rho_{2} \hat{W}\right\}_{k^{\prime \prime} q^{\prime \prime}}^{(C)} \equiv \sum_{s^{\prime \prime}, l^{\prime \prime}=1}^{M_{C}} \rho_{k^{\prime \prime} s^{\prime \prime} l^{\prime \prime} q^{\prime \prime}}^{(C)} \hat{W}_{s^{\prime \prime} l^{\prime \prime}}^{(C)}+\sum_{k, q=1}^{M_{A}} \rho_{k k^{\prime \prime} q q^{\prime \prime}}^{(A C)} \hat{W}_{k q}^{(C A)}+\sum_{k^{\prime}, q^{\prime}=1}^{M_{B}} \rho_{k^{\prime} k^{\prime \prime} q^{\prime} q^{\prime \prime}}^{(B C)} \hat{W}_{k^{\prime} q^{\prime}}^{(C B)}, \\
& \left\{\rho_{3} \hat{U}\right\}_{k^{\prime \prime} q^{\prime \prime}}^{(C)} \equiv \frac{1}{2} \sum_{s^{\prime \prime}, p^{\prime \prime}, r^{\prime \prime}, l^{\prime \prime}=1}^{M_{C}} \rho_{k^{\prime \prime} s^{\prime \prime} p^{\prime \prime} r^{\prime \prime} l^{\prime \prime} q^{\prime \prime}}^{(C)} \hat{U}_{s^{\prime \prime} p^{\prime \prime} l^{\prime \prime} r^{\prime \prime}}^{(C)}+\sum_{k, s, q, l=1}^{M_{A}} \rho_{k k^{\prime \prime} s l q q^{\prime \prime}}^{(A A C)} \hat{U}_{k s q l}^{(C A A)}+ \\
& \sum_{k, s^{\prime \prime}, q, l^{\prime \prime}=1}^{M_{A}, M_{C}} \rho_{k k^{\prime \prime} s^{\prime \prime} l^{\prime \prime} q q^{\prime \prime}}^{(A C C)} U_{k s^{\prime \prime} q l^{\prime \prime}}^{(C A C)}+\sum_{k^{\prime}, s^{\prime}, q^{\prime}, l^{\prime}=1}^{M_{B}} \rho_{k^{\prime} k^{\prime \prime} s^{\prime} l^{\prime} q^{\prime} q^{\prime \prime}}^{(B B C)} \hat{U}_{k^{\prime} s^{\prime} q^{\prime} l^{\prime}}^{(C B B)}+ \\
& \sum_{M_{B}, M_{C}}^{M^{\prime}, s^{\prime \prime}, q^{\prime}, l^{\prime \prime}=1} \rho_{k^{\prime} k^{\prime \prime} s^{\prime \prime} l^{\prime \prime} q^{\prime} q^{\prime \prime}}^{(B C C)} \hat{U}_{k^{\prime} s^{\prime \prime} q^{\prime} l^{\prime \prime}}^{(C B C)}+\sum_{k, k^{\prime}, q, q^{\prime}=1}^{M_{A}, M_{B}} \rho_{k k^{\prime} k^{\prime \prime} q q^{\prime} q^{\prime \prime}}^{(A B C)} \hat{U}_{k k^{\prime} q q^{\prime}}^{(C A B)} .
\end{aligned}
$$

These auxiliary one-body operators are constructed from products of matrix elements of reduced density matrices of increasing order (see Appendix B) times the one-body potentials resulting from interactions of the same order, see Eqs. (C4) and (C5). The derivation of the equations-of-motion (51) is now fully completed. 
[1] Time-Dependent Methods for Quantum Dynamics, edited by K. C. Kulander (North-Holland, Amsterdam, 1991).

[2] J. E. Bayfield, Quantum Evolution: An Introduction to Time-Dependent Quantum Mechanics (Wiley, New York, 1999).

[3] P. Ring and P. Schuck, The Nuclear Many-Body Problem (Springer, Berlin, 2000).

[4] Many-Particle Quantum Dynamics in Atomic and Molecular Fragmentation, edited by J. Ullrich and V. P. Shevelko (Springer, Berlin, 2003).

[5] L. Pitaevskii and S. Stringari, Bose-Einstein Condensation (Oxford University Press, Oxford, 2003).

[6] Quantum Dynamics of Complex Molecular Systems, edited by D. A. Micha and I. Burghardt, Springer Series in Chemical Physics, Vol. 83 (Springer, Berlin, 2007).

[7] H.-D. Meyer, U. Manthe, and L. S. Cederbaum, Chem. Phys. Lett. 165, 73 (1990).

[8] U. Manthe, H.-D. Meyer, and L. S. Cederbaum, J. Chem. Phys. 97, 3199 (1992).

[9] M. H. Beck, A. Jäckle, G. A. Worth, and H.-D. Meyer, Phys. Rep. 324, 1 (2000).

[10] Multidimensional Quantum Dynamics: MCTDH Theory and Applications, edited by H.-D. Meyer, F. Gatti, and G. A. Worth (Wiley-VCH, Weinheim, 2009).

[11] G. A. Worth, H.-D. Meyer, and L. S. Cederbaum, J. Chem. Phys. 109, 3518 (1998).

[12] A. Raab, G. Worth, H.-D. Meyer, and L. S. Cederbaum, J. Chem. Phys. 110, 936 (1999).

[13] R. van Harrevelt and U. Manthe, J. Chem. Phys. 123, 064106 (2005).

[14] L. S. Cederbaum, E. Gindensperger, and I. Burghardt, Phys. Rev. Lett. 94, 113003 (2005).

[15] H.-D. Meyer, F. Le Quere, C. Leonard, and F. Gatti, Chem. Phys. 329, 179 (2006).

[16] O. Vendrell, F. Gatti, D. Lauvergnat, and H.-D. Meyer, J. Chem. Phys. 127, 184302 (2007).

[17] O. Vendrell, F. Gatti, and H.-D. Meyer, J. Chem. Phys. 127, 184303 (2007).

[18] H. Tamura, J. G. S. Ramon, E. R. Bittner, and I. Burghardt, Phys. Rev. Lett. 100, 107402 (2008).

[19] H. Wang and M. Thoss, J. Chem. Phys. 119, 1289 (2003).

[20] U. Manthe, J. Chem. Phys. 128, 164116 (2008).

[21] O. Vendrell and H.-D. Meyer, J. Chem. Phys. 134, 044135 (2011).

[22] S. Zöllner, H.-D. Meyer, and P. Schmelcher, Phys. Rev. A 74, 053612 (2006). 
[23] S. Zöllner, H.-D. Meyer, and P. Schmelcher, Phys. Rev. A 74, 063611 (2006).

[24] S. Zöllner, H.-D. Meyer, and P. Schmelcher, Phys. Rev. Lett. 100, 040401 (2008).

[25] S. Zöllner, H.-D. Meyer, and P. Schmelcher, Phys. Rev. A 78, 013629 (2008).

[26] A. U. J. Lode, A. I. Streltsov, O. E. Alon, H.-D. Meyer, and L. S. Cederbaum, J. Phys. B 42, 044018 (2009).

[27] S. Zöllner, G. M. Bruun, C. J. Pethick, and S. M. Reimann, Phys. Rev. Lett. 107, 035301 (2011).

[28] J. Zanghellini, M. Kitzler, C. Fabian, T. Brabec, and A. Scrinzi, Laser Phys. 13, 1064 (2003).

[29] T. Kato and H. Kono, Chem. Phys. Lett. 392, 533 (2004).

[30] M. Nest, T. Klamroth, and P. Saalfrank, J. Chem. Phys. 122, 124102 (2005).

[31] A. I. Streltsov, O. E. Alon, and L. S. Cederbaum, Phys. Rev. Lett. 99, 030402 (2007).

[32] O. E. Alon, A. I. Streltsov, and L. S. Cederbaum, Phys. Rev. A 77, 033613 (2008).

[33] M. Kitzler, J. Zanghellini, Ch. Jungreuthmayer, M. Smits, A. Scrinzi, and T. Brabec, Phys. Rev. A 70, 041401(R) (2004).

[34] J. Caillat, J. Zanghellini, M. Kitzler, O. Koch, W. Kreuzer, and A. Scrinzi, Phys. Rev. A 71, $012712(2005)$.

[35] Z. Zhang, C. F. Destefani, C. McDonald, and T. Brabec, Phys. Rev. B 72, 161309(R) (2005).

[36] G. Jordan, J. Caillat, C. Ede, and A. Scrinzi, J. Phys. B 39, S341 (2006).

[37] M. Nest, Phys. Rev. A 73, 023613 (2006).

[38] M. Nest, R. Padmanaban, and P. Saalfrank, J. Chem. Phys. 126, 214106 (2007).

[39] F. Remacle, M. Nest, and R. D. Levine, Phys. Rev. Lett. 99, 183902 (2007).

[40] S. Sukiasyan, C. McDonald, C. Destefani, M. Yu. Ivanov, and T. Brabec, Phys. Rev. Lett. 102, 223002 (2009).

[41] D. Hochstuhl and M. Bonitz, J. Chem. Phys. 134, 084106 (2011).

[42] M. Mundt and D. J. Tannor, New J. Phys. 11, 105038 (2009).

[43] A. I. Streltsov, O. E. Alon, and L. S. Cederbaum, Phys. Rev. Lett 100, 130401 (2008).

[44] K. Sakmann, A. I. Streltsov, O. E. Alon, and L. S. Cederbaum, Phys. Rev. Lett. 103, 220601 (2009).

[45] A. I. Streltsov, O. E. Alon, and L. S. Cederbaum, Phys. Rev. Lett. 106, 240401 (2011).

[46] J. Grond, J. Schmiedmayer, and U. Hohenester, Phys. Rev. A 79, 021603 (2009).

[47] J. Grond, G. von Winckel, J. Schmiedmayer, and U. Hohenester, Phys. Rev. A 80, 053625 
(2009).

[48] P.-O. Löwdin, Phys. Rev. 97, 1474 (1955).

[49] A. J. Coleman and V. I. Yukalov, Reduced Density Matrices: Coulson's Challenge (SpringerVerlag, New York, 2000).

[50] D. A. Mazziotti, Phys. Rev. Lett. 93, 213001 (2004).

[51] D. A. Mazziotti, Phys. Rev. Lett. 97, 143002 (2006).

[52] G. Gidofalvi and D. A. Mazziotti, Phys. Rev. A 74, 012501 (2006).

[53] Reduced-Density-Matrix Mechanics: with Application to Many-electron Atoms and Molecules, edited by D. A. Mazziotti, Advances in Chemical Physics, Vol. 134 (Wiley, New York, 2007).

[54] D. A. Mazziotti, J. Chem. Phys. 126, 184101 (2007).

[55] E. Kamarchik and D. A. Mazziotti, Phys. Rev. A 75, 013203 (2007).

[56] A. I. Streltsov, O. E. Alon, and L. S. Cederbaum, Phys. Rev. A 73, 063626 (2006).

[57] O. E. Alon, A. I. Streltsov, L. S. Cederbaum, J. Chem. Phys. 127, 154103 (2007).

[58] O. E. Alon, A. I. Streltsov, and L. S. Cederbaum, Phys. Rev. A 76, 062501 (2007).

[59] O. E. Alon, A. I. Streltsov, and L. S. Cederbaum, Phys. Rev. A 79, 022503 (2009).

[60] A. I. Streltsov, O. E. Alon, and L. S. Cederbaum, Phys. Rev. A 81, 022124 (2010).

[61] A. I. Streltsov, K. Sakmann, O. E. Alon, and L. S. Cederbaum, Phys. Rev. A 83, 043604 (2011).

[62] A. I. Streltsov, K. Sakmann, A. U. J. Lode, O. E. Alon, and L. S. Cederbaum, The Multiconfigurational Time-Dependent Hartree for Bosons Package, Version 2.1, Heidelberg (2011); See [http://mctdhb.uni-hd.de].

[63] B. A. Loiseau and Y. Nogami, Nucl. Phys. B2, 470 (1967).

[64] J. Carlson, V. R. Pandharipande, and R. B. Wiringa, Nucl. Phys. A401, 59 (1983).

[65] H. P. Büchler, A. Micheli, P. Zoller, Nature Phys. 3, 726 (2007).

[66] T. Fukuhara1, S. Sugawa, and Y. Takahashi, Phys. Rev. A 76, 051604(R) (2007).

[67] M. Taglieber, A.-C. Voigt, T. Aoki, T. W. Hänsch, and K. Dieckmann, Phys. Rev. Lett. 100, 010401 (2008).

[68] C.-H. Wu, I. Santiago, J. W. Park, P. Ahmadi, and M. W. Zwierlein, Phys. Rev. A 84, 011601(R) (2011).

[69] P. Kramer and M. Saracento, Geometry of the time-dependent variational principle (Springer, Berlin, 1981). 
[70] H.-J. Kull and D. Pfirsch, Phys. Rev. E 61, 5940 (2000).

[71] P. A. M. Dirac, Proc. Cambridge Phil. Soc. 26, 376 (1930).

[72] J. Frenkel, Wave Mechanics (Oxford University Press, Oxford, 1934).

[73] A. P. J. Jansen, J. Chem. Phys. 99, 4051 (1993).

[74] U. Manthe, J. Chem. Phys. 101, 2652 (1994).

[75] T. Jun Park and J. C. Light, J. Chem. Phys. 85, 5870 (1986).

[76] A. Szabo and N. S. Ostlund, Modern Quantum Chemistry (Dover, Mineola, NY, 1996).

[77] Modern Electronic Structure Theory, edited by D. R. Yarkony, Advanced Series in Physical Chemistry, Vol. 2 (World Scientific, Singapore, 1995). 